\title{
HOMOLOGY OF AFFINE SPRINGER FIBERS IN THE UNRAMIFIED CASE
}

MARK GORESKY, ROBERT KOTTWITZ, and ROBERT MACPHERSON

\begin{abstract}
Assuming a certain "purity" conjecture, we derive a formula for the (complex) cohomology groups of the affine Springer fiber corresponding to any unramified regular semisimple element. We use this calculation to present a complex analog of the fundamental lemma for function fields. We show that the "kappa" orbital integral that arises in the fundamental lemma is equal to the Lefschetz trace of the Frobenius acting on the étale cohomology of a related variety.
\end{abstract}

\section{Contents}

1. Introduction . . . . . . . . . . . . . . . . . . . . . . . . 510

2. Purity for ind-varieties . . . . . . . . . . . . . . . . . . . . . . . . . . 512

3. Symmetric algebras . . . . . . . . . . . . . . . . . . . . . 516

4. Equivariant homology . . . . . . . . . . . . . . . . . . . . . . . . . . 518

5. Affine Springer fibers . . . . . . . . . . . . . . . . . . . . . 520

6. Affine Springer fibers for SL(2) . . . . . . . . . . . . . . . . . . . . . 525

7. Equivariant homology of SL(2) Springer fibers . . . . . . . . . . . . . . 527

8. Groups of semisimple rank one . . . . . . . . . . . . . . . . . . . . . . . 529

9. Equivariant homology of affine Springer fibers . . . . . . . . . . . . . . 531

10. Endoscopic groups . . . . . . . . . . . . . . . . . . . . . . . . . 534

11. The quotient under $\Lambda$. . . . . . . . . . . . . . . . . . . . . . . 538

12. Combinatorial lemmas . . . . . . . . . . . . . . . . . . . . . . 539

13. Affine flag manifold for SL(2) . . . . . . . . . . . . . . . . . . . 543

14. Homology of affine Springer fibers in the affine flag manifold . . . . . . . 547

15. An orbital integral . . . . . . . . . . . . . . . . . . . . . . . . . 550

References .. . . . . . . . . . . . . . . . . . . . 559

DUKE MATHEMATICAL JOURNAL

Vol. 121, No. 3, (C) 2004

Received 11 November 2002. Revision received 14 March 2003.

2000 Mathematics Subject Classification. Primary 14M15, 11D88, 11F85; Secondary 55N91.

Goresky's work partially supported by National Science Foundation grant numbers DMS-9900324 and DMS0139986.

Kottwitz's work partially supported by National Science Foundation grant number DMS-0071971. 


\section{Introduction}

Let $F=\mathbb{C}((\varepsilon))$ be the field of formal Laurent series, and let $\mathfrak{o}=\mathbb{C}[[\varepsilon]]$ be its integer ring of formal power series. A fundamental object of study in modern representation theory is the affine Grassmannian $X=G(F) / G(\mathfrak{o})$ associated to a complex reductive algebraic group $G$. Let $\mathfrak{g}=\operatorname{Lie}(G)$. Each regular semisimple element $\gamma \in \mathfrak{g}(\mathfrak{o})$ determines a "vector field" on $X$ whose fixed point set

$$
X_{\gamma}=\left\{x G(\mathfrak{o}) \in X: \operatorname{Ad}\left(x^{-1}\right)(\gamma) \in \mathfrak{g}(\mathfrak{o})\right\}
$$

is a finite-dimensional increasing union of complex projective varieties, which is known as the affine Springer fiber corresponding to $\gamma$. The study of affine Springer fibers was initiated in [KL2], where many of the basic properties of these varieties were described.

In this paper we suppose that $T \subset G$ is a maximal torus defined over $\mathbb{C}$, and we consider the affine Springer fiber $X_{\gamma}$ associated to regular semisimple elements $\gamma \in \mathfrak{t}(\mathfrak{o})$, where $\mathfrak{t}=\operatorname{Lie}(T)$. (Not all affine Springer fibers are of this type; since $T$ is split over $F$, we refer to this as the unramified case.) The complex torus $T(\mathbb{C})$ acts on $X_{\gamma}$. In Theorem 9.2, the first principal result of this paper, we give an explicit description of the $T\left(\mathbb{C}\right.$ )-equivariant homology of $X_{\gamma}$ under the assumption that the ordinary homology of $X_{\gamma}$ is pure, in the sense of mixed Hodge theory. (We conjecture that this always holds; cf. §5.3.) The description in $§ 9.2$ is made possible by using [GKM1, $\S 6.3]$ (the lemma of Chang and Skjelbred), which describes the equivariant homology of a $T(\mathbb{C})$-variety in terms of the 0 - and 1-dimensional orbits of $T(\mathbb{C})$. The computation is reduced to the case of the equivariant homology of affine Springer fibers for the group SL(2). Among the various ways to compute the equivariant homology in this special case of SL(2), we have chosen to describe one that is conceptually simple but computationally rather cumbersome. It uses the existence of a slightly larger torus $\widetilde{T}(\mathbb{C})$ which acts on this Springer fiber. To facilitate the exposition we have extracted the combinatorial details and placed them in $\S 12$.

Suppose that $G$ is adjoint, $s \in \widehat{T}$ is an element of the dual torus, and $(H, s)$ is endoscopic data for $G$. Then $H$ and $G$ share the same torus $T$, so $\gamma$ corresponds to a regular semisimple element $\gamma_{H} \in H$, and there is an associated affine Springer fiber $X_{\gamma_{H}}^{H}$. Motivated by the fundamental lemma ([L, §III.1]), one predicts that there should be a close relation between the homology groups $H_{*}\left(X_{\gamma} ; \mathbb{C}\right)$ and $H_{*}\left(X_{\gamma_{H}}^{H} ; \mathbb{C}\right)$. However, from a geometrical point of view, any relationship between these homology groups comes as a surprise since the group $H$ may not be a subgroup of $G$. In fact, the varieties $X_{\gamma}$ and $X_{\gamma_{H}}^{H}$ have very little to do with each other: there does not appear to be any map or correspondence between them, and even their dimensions are different, in general. Moreover, the relation between these homology groups turns out to be rather subtle: they are not simply isomorphic, but rather, they become isomorphic only after a degree shift and a certain localization. 
The element $s$ gives rise to a homomorphism $\chi_{*}(T) \rightarrow \mathbb{C}^{\times}$(defined on the cocharacter group of $T$ ) such that the set of coroots for $H$ is given by

$$
\Phi^{\vee}(H, T)=\left\{\alpha^{\vee} \in \Phi^{\vee}(G, T): s\left(\alpha^{\vee}\right)=1\right\} .
$$

The same cocharacter group $\chi_{*}(T)$ acts freely on $X_{\gamma}$. Let $J$ be the multiplicative subset of the group algebra $\mathbb{C}\left[\chi_{*}(T)\right]$ which is generated by the elements $\left(1-\alpha^{\vee}\right)$, where $\alpha^{\vee} \in \Phi^{\vee}(G, T)$ and $s\left(\alpha^{\vee}\right) \neq 1$. For any $\mathbb{C}\left[\chi_{*}(T)\right]$-module $V$, let $V_{J}=J^{-1} V$ be its localization with respect to $J$. Let $r=r(\gamma)$ be the nonnegative integer defined in $\$ 10.1$. The second principal result in this paper is the following complex analog to the fundamental lemma in the unramified case (a more precise version of which is stated in Theorem 10.2).

\section{THEOREM 1.1}

Suppose that the homology of $X_{\gamma}$ is pure and that the homology of $X_{\gamma_{H}}^{H}$ is pure. Then for each $i$ there is a homomorphism

$$
H_{i}^{T(\mathbb{C})}\left(X_{\gamma}, \mathbb{C}\right) \rightarrow H_{i-2 r}^{T(\mathbb{C})}\left(X_{\gamma_{H}}^{H}, \mathbb{C}\right)
$$

which becomes an isomorphism

$$
H_{i}^{T(\mathbb{C})}\left(X_{\gamma} ; \mathbb{C}\right)_{J} \cong H_{i-2 r}^{T(\mathbb{C})}\left(X_{\gamma_{H}}^{H} ; \mathbb{C}\right)_{J}
$$

after localizing with respect to $J$.

The homomorphism (1.1.1) is compatible with a number of algebras which act on these homology groups. First, the equivariant homology is a module over the equivariant cohomology $\mathscr{D}=H_{T(\mathbb{C})}^{*}(\mathrm{pt})$ of a point $(\S 4)$. Second, the group algebra $\mathbb{C}\left[\chi_{*}(T)\right]$ acts. Finally, a certain group $\widetilde{\mathscr{W}}_{\gamma}^{G, H}$ of automorphisms of the situation also acts (cf. $\S \S 9.4$ and 10.1), and the homomorphism (1.1.1) transforms by a certain character $\eta$ under $\widetilde{\mathscr{W}}_{\gamma}^{G, H}$.

Using the action of $\mathscr{D}$, it is possible to recover the ordinary homology of $X_{\gamma}$ from its equivariant homology, so (1.1.1) gives a homomorphism

$$
H_{i}\left(X_{\gamma} ; \mathbb{C}\right) \rightarrow H_{i-2 r}\left(X_{\gamma_{H}}^{H} ; \mathbb{C}\right)
$$

of the ordinary homology, which also becomes an isomorphism after localizing with respect to $J$. In $\S 10$ the action of the group algebra $\mathbb{C}\left[\chi_{*}(T)\right]$ is used in order to (partially) describe the homology of the quotient $\chi_{*}(T) \backslash X_{\gamma}$. Finally, the action of the group $\widetilde{\mathscr{W}}_{\gamma}^{G, H}$ provides a complex analog for the action of the Frobenius automorphism.

These extra ingredients are related to the fundamental lemma for function fields. In Theorem 15.8, the $\kappa$-orbital integral that occurs in the fundamental lemma is shown 
to equal the trace of the Frobenius automorphism on the étale cohomology of the quotient $\chi_{*}(T) \backslash X_{\gamma}(\bar{k})$, where now $X_{\gamma}(\bar{k})$ is the finite characteristic analog of the complex algebraic variety $X_{\gamma}$. Next, the analogs of Theorems 9.2 and 10.2 need to be established in étale homology, a task that we have not fully carried out here. In $\$ 15.12$ we indicate how these results are related to the fundamental lemma in the unramified case.

We would also like to draw attention to the recent preprint [La2], in which G. Laumon uses the methods and techniques of the present paper together with a deformation argument from [La1] to prove (under the same purity assumption) the "geometric fundamental lemma" (i.e., the étale cohomology analog of Proposition 11.2) for unitary groups (and for arbitrary regular semisimple elements $\gamma$ ).

In $\S \S 13$ and 14 we list the changes that are needed in order to establish similar results for Springer fibers $Y_{\gamma}$ in the affine flag manifold $Y$. The homology of a Springer fiber in the affine flag manifold carries the additional structure of a (right) action of the affine Weyl group, the Springer action. It was constructed by Lusztig [Lu] and (using a statement from [KL1] whose proof does not appear in the literature) by Sage [S1]. In $§ 14.4$ this action is explicitly described using our formula (Theorem 14.3) for the homology of the Springer fiber $Y_{\gamma}$ (under the assumption that this homology is pure). In Theorem 14.6 we state the main consequence: If $(H, s)$ is endoscopic data for $G$, if $H_{*}\left(Y_{\gamma} ; \mathbb{C}\right)$ is pure, and if $H_{*}\left(Y_{\gamma_{H}}^{H} ; \mathbb{C}\right)$ is pure, then for each $i$ we obtain a homomorphism

$$
H_{i}^{T(\mathbb{C})}\left(Y_{\gamma} ; \mathbb{C}\right) \rightarrow H_{i-2 r}^{T(\mathbb{C})}\left(Y_{\gamma_{H}}^{H} ; \mathbb{C}\right) \otimes_{\mathbb{C}\left[\widetilde{W}^{H}\right]} \mathbb{C}[\widetilde{W}]
$$

of $\mathscr{D}$-modules, and a homomorphism on ordinary homology,

$$
H_{i}\left(Y_{\gamma} ; \mathbb{C}\right) \rightarrow H_{i-2 r}\left(Y_{\gamma_{H}}^{H} ; \mathbb{C}\right) \otimes_{\mathbb{C}\left[\widetilde{W}^{H}\right]} \mathbb{C}[\widetilde{W}],
$$

each of which is equivariant with respect to the right action of $\widetilde{W}$ and transforms by the character $\eta$ under the action of $\widetilde{\mathscr{W}}_{\gamma}^{G, H}$. Each of these homomorphisms becomes an isomorphism after localizing with respect to $J$.

\section{Purity for ind-varieties}

\section{1}

Let $X$ be a complex projective algebraic variety with (increasing) weight filtration $W$ of the cohomology $H^{*}=H^{*}(X ; \mathbb{C})$ (see [D2]). The cohomology of $X$ is pure if

$$
\mathrm{Gr}^{m} W\left(H^{i}\right)=W^{m}\left(H^{i}\right) / W^{m-1}\left(H^{i}\right)=0 \quad \text { for } i \neq m .
$$

We obtain an increasing weight filtration on the homology $H_{*}=H_{*}(X ; \mathbb{C})$ :

$$
W^{m}\left(H_{i}\right)=\left(H^{i} / W^{-1-m}\left(H^{i}\right)\right)^{*}
$$


by duality. If $f: X \rightarrow Y$ is a morphism of projective varieties, the induced mapping $f_{*}: H_{*}(X) \rightarrow H_{*}(Y)$ is strictly compatible with the weight filtration.

Let $X$ be a complex projective ind-variety (see [Ku], [Sh]), that is, a set with a filtration $X_{0} \subset X_{1} \subset \cdots$ by complex projective varieties such that $X=\bigcup_{n=1}^{\infty} X_{n}$ and such that each $X_{n} \rightarrow X_{n+1}$ is a closed immersion. A set $Y \subset X$ is closed in the limit topology for $X$ if and only if $Y \cap X_{n}$ is closed (in the classical topology) for each $n$. Then $H_{*}(X)=\lim _{\rightarrow} H_{*}\left(X_{n}\right)$ inherits a limit weight filtration $W$ from the weight filtrations on $H_{*}\left(X_{n}\right)$. If $X=\bigcup_{n=1}^{\infty} Y_{n}$ is an equivalent ind-variety structure on $X$ (meaning that the identity mapping $X \rightarrow X$ is a morphism of $i n d$-varieties), then the resulting weight filtration $W^{\prime}$ on $H_{*}(X)$ agrees with $W$. We say that the homology of $X$ is pure if $\mathrm{Gr}^{-m} W\left(H_{i}(X)\right)=0$ for $m \neq i$.

Let $A=\left(\mathbb{C}^{\times}\right)^{m}$ be a complex torus that acts on a projective ind-variety $X$ such that each $X_{n}$ is invariant under $A$ and such that the action of $A$ on $X_{n}$ is algebraic and is compatible with the immersion $X_{n} \rightarrow X_{n+1}$. In this case we say that $A$ acts algebraically on $X$. The classifying space $B A$ may be taken to be the ind-variety $\left(\mathbf{P}^{1}\right)^{m} \subset\left(\mathbf{P}^{2}\right)^{m} \subset \cdots$, which has pure homology. The Cartan-Leray spectral sequence for the $A$-equivariant homology of $X_{n}$ is the spectral sequence for the fibration $X_{n} \times{ }_{A} E A \rightarrow B A$ with $E^{2} \cong H_{*}(B A) \otimes H_{*}\left(X_{n}\right)$. It inherits a weight filtration such that the differentials are strictly compatible with the weight. It follows that the CartanLeray spectral sequence for the $A$-equivariant homology of $X$ inherits a weight filtration such that the differentials are strictly compatible with the weight. The following fact is needed for Corollary 10.3.

\section{LEMMA 2.2}

Suppose that $X$ is a complex projective ind-variety with an action of an algebraic torus A. Suppose that the homology of $X$ is pure. Then the homology of $X$ is equivariantly formal (see [GKMI, \$1]); that is, the Cartan-Leray spectral sequence for the A-equivariant homology of $X$ collapses at $E^{2}$ and gives rise to an isomorphism

$$
H_{*}^{A}(X ; \mathbb{C}) \cong H_{*}(X ; \mathbb{C}) \otimes_{\mathbb{C}} H_{*}^{A}(\mathrm{pt} ; \mathbb{C}) .
$$

\subsection{Purity of $B \mathbb{Z}^{n}$}

Define an action of the additive group $\mathbb{Z}$ on the contractible ind-variety

$$
E \mathbb{Z}=(\mathbb{Z} \times \mathbb{C}) /(m, 0) \sim(m+1,1)
$$

(which is a countable union of affine lines joined each to the next at a single point) by $m \cdot(r, t)=(r+2 m, t)$ (where $m, r \in \mathbb{Z}$ and $t \in \mathbb{C}$ ). The quotient $B \mathbb{Z}=E \mathbb{Z} / \mathbb{Z}$ consists of two copies $V_{0}, V_{1}$ of $\mathbb{C}$, joined at two points: $0 \in V_{0}$ is identified with 
$1 \in V_{1}$, and vice versa. The inclusion of the unit circle $T^{1} \rightarrow B \mathbb{Z}$ given by

$$
e^{i \theta} \mapsto \begin{cases}\frac{\theta}{\pi} \in V_{0} & \text { if } 0 \leq \theta \leq \pi, \\ \frac{\theta}{\pi}-1 \in V_{1} & \text { if } \pi \leq \theta \leq 2 \pi\end{cases}
$$

is a homotopy equivalence. Let $\Lambda=\mathbb{Z}^{n}$, and set $E \Lambda=(E \mathbb{Z})^{n}$ and $B \Lambda=(B \mathbb{Z})^{n}$. We obtain a homotopy equivalence $T^{n}=\left(T^{1}\right)^{n} \rightarrow B \Lambda$.

Suppose that $X$ is an ind-variety on which $\Lambda$ acts freely by morphisms. Let $E(X)=E \Lambda \times_{\Lambda} X$ be the Borel construction, together with its projections

$$
\Lambda \backslash X \stackrel{p}{\longleftarrow} E(X) \stackrel{\pi}{\longrightarrow} B \Lambda
$$

Let $s: \Lambda \rightarrow \mathbf{G L}(1, \mathbb{C})$ be a 1-dimensional representation of finite order, and write $\mathbb{C}_{s}$ for the representation space. Then $s$ determines local systems $\mathscr{L}_{s}=\mathbb{C}_{s} \times{ }_{\Lambda} X$ on $\Lambda \backslash X$ and $\mathscr{L}_{s}^{B}=E \Lambda \times{ }_{\Lambda} \mathbb{C}_{s}$ on $B \Lambda$, and there is a canonical isomorphism of local systems, $\pi^{*} \mathscr{L}_{s}^{B} \cong p^{*} \mathscr{L}_{s}$. Denote this local system on $E(X)$ by $\mathscr{L}_{s}^{E}$. The homology Leray spectral sequence, with coefficients in $\mathscr{L}_{s}^{E}$, for the fibration $\pi: E(X) \rightarrow B \Lambda$ has

$$
E_{p q}^{2}=H_{p}\left(\Lambda ; H_{q}\left(X ; \mathbb{C}_{s}\right)\right)=H_{p}\left(B \Lambda ; \mathscr{L}_{s}^{B} \otimes \mathbf{H}_{\mathbf{q}}\right) \Longrightarrow H_{p+q}\left(\Lambda \backslash X ; \mathscr{L}_{s}\right),
$$

where $\mathbf{H}_{\mathbf{q}}$ denotes the local system on $B \Lambda$ corresponding to $H_{q}(X ; \mathbb{C})$. The following fact is needed for Proposition 11.2.

\section{LEMMA 2.4}

Suppose that the homology of $X$ is pure. Then the spectral sequence (2.3.1) collapses at $E^{2}$. Hence there is an isomorphism

$$
H_{m}\left(\Lambda \backslash X ; \mathscr{L}_{s}\right) \cong \bigoplus_{p+q=m} H_{p}\left(\Lambda ; H_{q}\left(X ; \mathbb{C}_{s}\right)\right)
$$

\subsection{Proof}

First consider the case when $n=1$ and when $s$ is the trivial representation. The homology $H_{*}\left(B \mathbb{Z} ; \mathbf{H}_{\mathbf{q}}\right)$ may be computed from the Mayer Vietoris sequence for the covering of $B \mathbb{Z}$ by the two (Zariski) closed sets $V_{0}$ and $V_{1}$. It is easy to see that this sequence gives a short exact sequence (of mixed Hodge structures)

0

$$
H_{1}\left(B \mathbb{Z} ; \mathbf{H}_{\mathbf{q}}\right) \longrightarrow H_{q} \longrightarrow H_{q-1} H_{q}\left(B \mathbb{Z} ; \mathbf{H}_{\mathbf{q}}\right) \longrightarrow 0,
$$

where $H_{q}=H_{q}(X ; \mathbb{C})$ and where $\mu$ is the homomorphism induced on homology from the action of $1 \in \mathbb{Z}$.

Next consider the case of general $n$ but still with $s$ the trivial representation. It follows by induction that the homology $H_{*}\left(B \mathbb{Z}^{n} ; \mathbf{H}_{\mathbf{q}}\right)$ is the homology of the Koszul 
complex $K_{\bullet}=\bigwedge^{*}\left(\mathbb{C}^{n}\right) \otimes H_{q}$ with differential

$$
\partial\left(e_{i_{1}} \wedge \cdots \wedge e_{i_{r}} \otimes h\right)=\sum_{j=1}^{r}(-1)^{j+1} e_{i_{1}} \wedge \cdots \wedge \widehat{e}_{i_{j}} \wedge \cdots \wedge e_{i_{r}} \otimes\left(\mu_{i_{j}}(h)-h\right),
$$

where $\mu_{1}, \ldots, \mu_{n}$ are the homomorphisms on $H_{q}$ determined by the action of the standard $n$ basis elements $e_{1}, \ldots, e_{n}$. Each $\bigwedge^{k}\left(\mathbb{C}^{n}\right) \otimes H_{q}$ is pure of weight $-q$ since it arises as a direct sum of copies of $H_{q}$. So each homology group $H_{p}\left(B \Lambda ; \mathbf{H}_{\mathbf{q}}\right)$ is pure of weight $-q$. Since the differentials in the spectral sequence (2.3.1) are strictly compatible with the weight filtration, this implies that the spectral sequence collapses at $E^{2}$.

Now consider the case of a nontrivial representation $s$ of finite order. Such a representation factors through the (finite) quotient $\Lambda / N \Lambda$ for some positive integer $N$. Let $\phi: \widetilde{B}=E \Lambda / N \Lambda \rightarrow B \Lambda$ be the resulting (finite) cover, and let $\widetilde{E}$ be the fiber product in the diagram

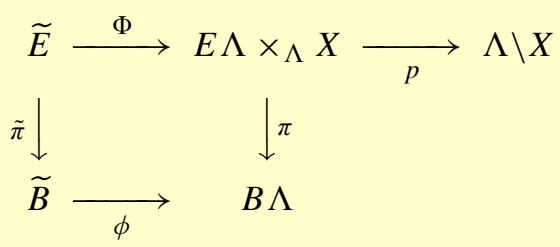

The preceding argument may be used to see that the Leray spectral sequence for the mapping $\tilde{\pi}$ (with constant coefficients) collapses at $E^{2}$.

The covering $\phi$ is a principal homogeneous space for the group $\Lambda / N \Lambda$, which acts by deck transformations on $\widetilde{B}$. So it acts on the local system $\phi_{*}(\mathbb{C})$ as the regular representation and determines decompositions

$$
\phi_{*}(\mathbb{C}) \cong \bigoplus_{\theta} \mathscr{L}_{\theta}^{B} \quad \text { and } \quad \Phi_{*}(\mathbb{C}) \cong \bigoplus_{\theta} \mathscr{L}_{\theta}^{E}
$$

into 1-dimensional local systems corresponding to the distinct characters $\theta$ : $\Lambda / N \Lambda \rightarrow \mathbb{C}^{\times}$. It follows (see below) that there is a natural isomorphism

$$
H_{*}\left(\widetilde{B} ; \mathbf{H}_{\mathbf{q}}^{\widetilde{\mathbf{B}}}\right) \cong \bigoplus_{\theta} H_{*}\left(B \Lambda ; \mathbf{H}_{\mathbf{q}} \otimes \mathscr{L}_{\theta}\right),
$$

where $\mathbf{H}_{\mathbf{q}}^{\widetilde{\mathbf{B}}}$ (resp., $\mathbf{H}_{\mathbf{q}}$ ) is the local system on $\widetilde{B}$ (resp., on $B \Lambda$ ) corresponding to $H_{q}(X ; \mathbb{C})$. In fact, the whole spectral sequence for $\tilde{\pi}$ decomposes under $\Lambda / N \Lambda$ into a direct sum (over distinct characters $\theta$ of $\Lambda / N \Lambda$ ) of spectral sequences for $\pi$ with coefficients in $\mathscr{L}_{\theta}$. It follows that each of these constituent spectral sequences, one of which corresponds to the character $s$, collapses.

There are several ways to verify equation (2.5.2). One way is to use the homotopy equivalence $T^{n} \rightarrow B \Lambda$, Poincaré duality, and (2.5.1). However, it may also be verified 
directly. Let $\mathbf{D}_{\mathbf{B} \Lambda}$ (resp., $\mathbf{D}_{\widetilde{\mathbf{B}}}$ ) be the dualizing complex on $B \Lambda$ (resp., on $\left.\widetilde{B}\right)$. Since $\phi$ is a finite covering space, $\phi_{*} \cong R \phi_{*} \cong R \phi_{\text {! }}$ and $\phi^{*}\left(\mathbf{D}_{\mathbf{B} \Lambda}\right) \cong \mathbf{D}_{\widetilde{B}}$. Since the diagram above is a fiber square,

$$
R \phi_{*}\left(\mathbf{H}_{q}^{\widetilde{B}}\right) \cong R \phi_{*}\left(\phi^{*} \mathbf{H}_{\mathbf{q}} \otimes \mathbb{C}\right) \cong \mathbf{H}_{\mathbf{q}} \otimes\left(\bigoplus_{\theta} \mathscr{L}_{\theta}^{B}\right) .
$$

The homology $H_{*}\left(\widetilde{B} ; \mathbf{H}_{\mathbf{q}}^{\widetilde{\mathbf{B}}}\right)$ is the cohomology (with compact supports) of the sheaf

$$
\mathbf{D}_{\widetilde{\mathbf{B}}} \otimes \mathbf{H}_{\mathbf{q}}^{\widetilde{\mathbf{B}}} \cong \phi^{*} \mathbf{D}_{\mathbf{B}} \otimes \mathbf{H}_{\mathbf{q}}^{\widetilde{\mathbf{B}}} .
$$

Pushing this sheaf forward under $\phi$ gives

$$
\mathbf{D}_{\mathbf{B}} \otimes R \phi_{*}\left(\mathbf{H}_{\mathbf{q}}^{\widetilde{\mathbf{B}}}\right) \cong \mathbf{D}_{\mathbf{B}} \otimes \mathbf{H}_{\mathbf{q}} \otimes\left(\bigoplus_{\theta} \mathscr{L}_{\theta}^{B}\right),
$$

whose compactly supported cohomology is $\bigoplus_{\theta} H_{*}\left(B \Lambda ; \mathbf{H}_{\mathbf{q}} \otimes \mathscr{L}_{\theta}^{B}\right)$, as claimed.

\subsection{Remarks}

Presumably the theory of weights can be extended to include (co)homology groups such as $H_{k}\left(B \Lambda ; \mathbf{H}_{\mathbf{q}} \otimes \mathscr{L}_{s}\right)$, in which case equation (2.5.2) implies that this group is pure of weight $-q$. We remark that if the homology of $X$ is both pure and Tate (meaning that it lives only in even degrees and that $G r^{-2 q} H_{*}(X)$ is all of Hodge type $(q, q)$ ), then the decomposition (2.4.1) is canonical because in this case the Hodge filtration and the weight filtration are opposed (see [D1, Prop. 1.2.5]). It seems likely that Lemma 2.4 remains valid even when the local system $s$ fails to have finite order.

\section{Symmetric algebras}

\section{1}

Consider the polynomial algebra

$$
\mathbf{S}=\mathbb{Q}\left[x_{1}, \ldots, x_{n}\right]=\bigoplus_{k=0}^{\infty} \mathbf{S}_{k}
$$

in $n$ variables, graded by degree (with finite-dimensional graded pieces), and consider its dual algebra of differential operators

$$
\mathscr{D}=\mathbb{Q}\left[\partial_{1}, \ldots, \partial_{n}\right]=\bigoplus_{k=0}^{\infty} \mathscr{D}_{k}
$$

with $\partial_{i} x_{j}=\delta_{i j}$. Then $\mathscr{D}_{k} \mathbf{S}_{j} \subset \mathbf{S}_{j-k}$. The natural pairing

$$
\mathscr{D} \otimes \mathbf{S} \stackrel{\langle\cdot \cdot \cdot\rangle}{\longrightarrow} \mathbb{Q}
$$


given by $\langle\partial, P\rangle=(\partial P)(0)$ satisfies

$$
\left\langle\partial \partial^{\prime}, P\right\rangle=\left\langle\partial, \partial^{\prime} P\right\rangle .
$$

It restricts to a nondegenerate (finite-dimensional) bilinear form $\mathscr{D}_{k} \otimes \mathbf{S}_{k} \rightarrow \mathbb{Q}$, which vanishes on $\mathscr{D}_{k} \otimes \mathbf{S}_{j}$ for $j \neq k$. If $I=\bigoplus_{k=0}^{\infty} I_{k} \subset \mathscr{D}$ is a homogeneous ideal, define

$$
\mathbf{S}\{I\}=\{s \in \mathbf{S}: \partial s=0 \text { for all } \partial \in I\}
$$

to be the subspace annihilated by $I$. It follows from (3.1.2) that

$$
\mathbf{S}\{I\}=I^{\perp}=\bigoplus_{k=0}^{\infty} I_{k}^{\perp},
$$

where

$$
I_{k}^{\perp}=\left\{s \in \mathbf{S}_{k}:\langle\partial, s\rangle=0 \text { for all } \partial \in I_{k}\right\} .
$$

So the pairing (3.1.1) passes to a nondegenerate pairing $\left(\mathscr{D}_{k} / I_{k}\right) \otimes I_{k}^{\perp} \rightarrow \mathbb{Q}$. If $\partial \in \mathscr{D}$, set $\mathbf{S}\{\partial\}=\mathbf{S}\{(\partial)\}$.

LEMMA 3.2

Suppose $\partial, \partial^{\prime} \in \mathscr{D}$ are relatively prime homogeneous elements of $\mathscr{D}$. Then

$$
\mathbf{S}\left\{\partial \partial^{\prime}\right\}=\mathbf{S}\{\partial\}+\mathbf{S}\left\{\partial^{\prime}\right\}
$$

and

$$
\mathbf{S}\{\partial\} \stackrel{\partial^{\prime} \cdot}{\longrightarrow} \mathbf{S}\{\partial\}
$$

is surjective and lowers degree by $\operatorname{deg}\left(\partial^{\prime}\right)$.

\subsection{Proof}

If $I, J \subset \mathscr{D}$ are homogeneous ideals, then it follows from (3.1.3) that

$$
\begin{aligned}
& \mathbf{S}\{I+J\}=\mathbf{S}\{I\} \cap \mathbf{S}\{J\}, \\
& \mathbf{S}\{I \cap J\}=\mathbf{S}\{I\}+\mathbf{S}\{J\} .
\end{aligned}
$$

Taking $I=(\partial)$ and $J=\left(\partial^{\prime}\right)$ proves (3.2.1). Since $\partial$ and $\partial^{\prime}$ are relatively prime, the mapping

$$
\mathscr{D} /(\partial) \stackrel{\partial^{\prime} \cdot}{\longrightarrow} \mathscr{D} /(\partial)
$$

is an injective homomorphism of graded algebras, which raises degree by $\operatorname{deg}\left(\partial^{\prime}\right)$. It follows by duality (3.1.1) that (3.2.2) is surjective. 


\section{Equivariant homology}

4.1

Let $A=A(\mathbb{C})$ be a complex torus with Lie algebra $\mathfrak{a}$. We often use without comment the canonical isomorphism $\chi^{*}(A) \otimes \mathbb{C} \rightarrow \mathfrak{a}^{*}$. Let

$$
\mathscr{D}(\mathfrak{a})=\operatorname{Sym}\left(\mathfrak{a}^{*}\right)=\bigoplus_{d=0}^{\infty} \operatorname{Sym}^{d}\left(\mathfrak{a}^{*}\right)
$$

be the symmetric algebra of linear differential operators (with constant complex coefficients) on $\mathfrak{a}^{*}$, graded by degree. Let

$$
\mathbf{S}(\mathfrak{a})=\operatorname{Sym}(\mathfrak{a})=\bigoplus_{d=0}^{\infty} \operatorname{Sym}^{d}\left(\mathfrak{a}^{*}\right)^{*}
$$

be the dual symmetric algebra of complex-valued polynomial functions on $\mathfrak{a}^{*}$.

Throughout this paper, cohomology and homology are taken with complex coefficients (unless otherwise stated); however, it is possible to use rational coefficients (e.g., by replacing $\mathfrak{a}^{*}$ with $\chi^{*}(A) \otimes \mathbb{Q}$ ). Let $B A$ be the classifying space for $A$. If $\varphi: A \rightarrow \mathbb{C}^{\times}$is a character, let $c_{1}(\varphi)$ be the first Chern class of the resulting line bundle on $B A$. Then $c_{1}$ extends to an isomorphism

$$
\mathscr{D}(\mathfrak{a}) \rightarrow H^{*}(B A)=H_{A}^{*}(\mathrm{pt})
$$

(the Chern-Weil isomorphism) of graded algebras, which doubles degrees.

Multiplication $A \times A \rightarrow A$ determines an H-space structure $m: B A \times B A \rightarrow$ $B A$ which gives rise to an algebra structure on $H_{*}(B A)=H_{*}^{A}(\mathrm{pt})$. The homology $H_{*}(B A)$ is also a module over $H^{*}(B A)$ under the cap product. If $x \in H^{2}(B A)$, then the Hopf formula $m^{*}(x)=1 \otimes x+x \otimes 1$ implies that $x$ acts on $H_{*}(B A)$ as a derivation, so $H^{*}(B A)$ acts on $H_{*}(B A)$ by differential operators. It follows that the mapping $\mathbf{S}(\mathfrak{a}) \rightarrow H_{*}(B A)$ (dual to the Chern-Weil isomorphism) is an algebra isomorphism and is compatible with the cap product, in the sense that the following diagram commutes:

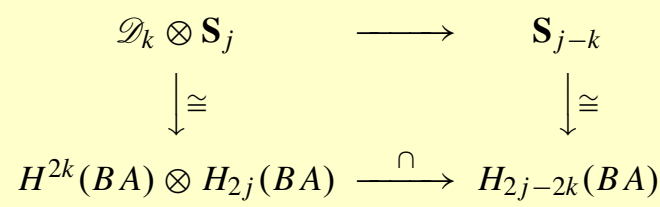

Suppose that $A$ acts on a complex projective algebraic variety $X$ whose cohomology is pure. Then the cohomology of $X$ is equivariantly formal and the Cartan-Leray spectral sequence for the equivariant cohomology of $X$ collapses at $E_{2}$. Let $T \subset A$ be 
a (possibly trivial) subtorus, and let $I=\operatorname{ker}\left(H^{*}(B A) \rightarrow H^{*}(B T)\right)$ be the resulting homogeneous ideal. Then the $T$-equivariant cohomology of $X$ may be recovered from the $A$-equivariant cohomology by

$$
H_{T}^{*}(X)=H_{A}^{*}(X) \otimes_{H_{A}^{*}(\mathrm{pt})} H_{T}^{*}(\mathrm{pt}) .
$$

The equivariant homology $H_{*}^{A}(X)$ is a module over $\mathscr{D}=H_{A}^{*}(\mathrm{pt})$, and by duality, the $T$-equivariant homology of $X$,

$$
H_{*}^{T}(X)=H_{*}^{A}(X)\{I\},
$$

is the submodule of $H_{*}^{A}(X)$ consisting of those elements that are annihilated by the homogeneous ideal $I$.

\section{2}

Let $\varphi: A \rightarrow \mathbb{C}^{\times}$be a nontrivial character with corresponding differential operator $\partial_{\varphi} \in \mathscr{D}(\mathfrak{a})$. Let $M=\operatorname{ker}(\varphi)$ with Lie algebra $\mathfrak{m}=\operatorname{ker}\left(\partial_{\varphi}\right) \subset \mathfrak{a}^{*}$. Let $j^{*}: \mathbf{S}(\mathfrak{m}) \rightarrow \mathbf{S}(\mathfrak{a})$ be the homomorphism induced by inclusion $\mathfrak{m} \subset \mathfrak{a}$. Then the image of $j^{*}$ consists of the polynomial functions $\mathbf{S}(\mathfrak{a})\left\{\partial_{\varphi}\right\}$ on $\mathfrak{a}^{*}$ which are annihilated by $\partial_{\varphi}$.

Such a nontrivial character $\varphi$ extends to an action of $A$ on $\mathbb{C P}^{1}=\mathbb{C}^{\times} \cup\{0\} \cup\{\infty\}$. As in $[G K M 1, \S \S 7.1,7.2]$, the long exact sequence for the pair $\left(\mathbb{C P}^{1},\{0\} \cup\{\infty\}\right)$ breaks into short exact sequences

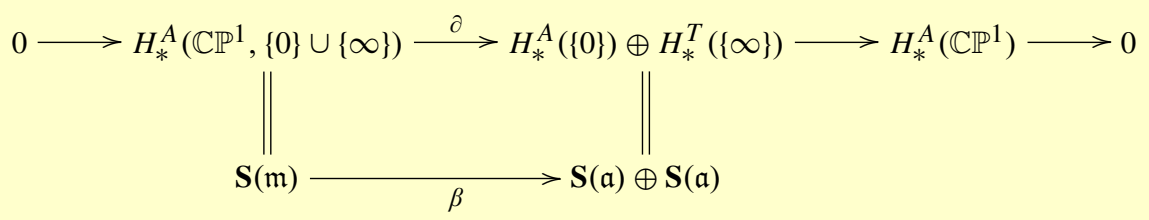

where $\beta(f)=\left(j^{*}(f),-j^{*}(f)\right)$.

4.3

Now suppose that a complex algebraic torus $A$ acts algebraically on a complex projective variety $Y$ whose cohomology is pure. Let $Y_{0} \subset Y$ be the set of fixed points, and let $Y_{1} \subset Y$ be the union of the 0 - and 1-dimensional orbits of $A$ in $Y$. The lemma of Chang and Skjelbred [CS, Lemma 2.3] (cf. [GKM1, §6.3]), when translated into homology, says that the following sequence is exact:

$$
H_{*}^{A}\left(Y_{1}, Y_{0}\right) \stackrel{\partial}{\longrightarrow} H_{*}^{A}\left(Y_{0}\right) \longrightarrow H_{*}^{A}(Y) \longrightarrow 0 .
$$

Suppose, moreover, that there are finitely many fixed points $Y_{0}=\left\{y_{1}, y_{2}, \ldots, y_{r}\right\}$ and finitely many 1-dimensional orbits $\left\{E_{1}, E_{2}, \ldots, E_{d}\right\}$. The closure $\bar{E}_{i}$ of each 1dimensional orbit is obtained by adding two fixed points $\partial E_{i}=\left\{y_{i_{a}}\right\} \cup\left\{y_{i_{b}}\right\}$. Let 
$\mathfrak{m}_{i} \subset \mathfrak{a}$ be the Lie algebra of the stabilizer of any point in $E_{i}$, and let $j_{i}^{*}: \mathbf{S}\left(\mathfrak{m}_{i}\right) \rightarrow$ $\mathbf{S}(\mathfrak{a})$ be the resulting homomorphism. Using $\S 4.2$, the sequence (4.3.1) becomes

$$
\bigoplus_{i=1}^{d} \mathbf{S}\left(\mathfrak{m}_{i}\right) \longrightarrow \bigoplus_{k=1}^{r} \mathbf{S}(\mathfrak{a}) \longrightarrow H_{*}^{A}(Y) \longrightarrow 0,
$$

where $\beta=\sum_{i=1}^{d} \beta_{i}$ with

$$
\beta_{i}\left(f_{i}\right)=\left(0, \ldots, j_{i}^{*}\left(f_{i}\right), \ldots,-j_{i}^{*}\left(f_{i}\right), \ldots, 0\right)
$$

for any $f_{i} \in \mathbf{S}\left(\mathfrak{m}_{i}\right)$. (Here, the two nonzero entries occur in the positions corresponding to $y_{i_{a}}$ and $y_{i_{b}}$, resp.)

\section{Affine Springer fibers}

\subsection{Notation}

Let $\mathbb{G}_{m}$ denote the multiplicative group. If $k$ is a field, if $\bar{k}$ is an algebraic closure, and if $T$ is an algebraic torus defined over $k$, let $\chi_{*}(T)=\operatorname{Hom}\left(\mathbb{G}_{m}, T\right) \cong \operatorname{Hom}\left(\bar{k}^{\times}, T(\bar{k})\right)$ denote the group of cocharacters of $T$, and let $\chi^{*}(T)=\operatorname{Hom}\left(T, \mathbb{G}_{m}\right) \cong$ $\operatorname{Hom}\left(T(\bar{k}), \bar{k}^{\times}\right)$denote the group of characters of $T$. The dual torus is $\widehat{T}=$ $\operatorname{Hom}\left(\chi_{*}(T), \mathbb{G}_{m}\right)$. If $G$ is a connected reductive complex algebraic group, if $T \subset G$ is a maximal torus in $G$, and if $\left(\chi^{*}(T), \Phi, \chi_{*}(T), \Phi^{\vee}\right)$ is the resulting root datum for $G$ (where $\Phi$ and $\Phi^{\vee}$ are the roots and coroots of $T$ in $G$, resp.), then we denote by $\widehat{G}$ the "dual" connected reductive complex algebraic group corresponding to the root datum $\left(\chi_{*}(T), \Phi^{\vee}, \chi^{*}(T), \Phi\right)$.

Throughout this paper (except in $\S 15$ ), we let $F=\mathbb{C}((\varepsilon))$ be the field of formal Laurent series over $\mathbb{C}$ and denote by $\mathfrak{o}=\mathbb{C}[[\varepsilon]]$ its ring of integers, the formal power series over $\mathbb{C}$. The valuation val $: F \rightarrow \mathbb{Z}$ takes integer values. Let $T$ be an algebraic torus defined over $\mathbb{C}$. The following sequence is exact:

$$
1 \longrightarrow T(\mathfrak{o}) \longrightarrow T(F) \stackrel{\text { val }}{\longrightarrow} \chi_{*}(T) \longrightarrow 1 \text {. }
$$

Here, val is defined by the property that $\alpha(\operatorname{val}(\ell))=\operatorname{val}(\alpha(\ell))$ for all $\alpha \in \chi^{*}(T)$ and all $\ell \in T(F)$. (On the left side of this equality, $\alpha$ is viewed as a homomorphism $\chi_{*}(T) \rightarrow \mathbb{Z}$.) The choice of uniformizing parameter $\varepsilon$ determines a splitting $\chi_{*}(T) \rightarrow T(F)$ by $\beta \mapsto \beta(\varepsilon)$, whose image $\Lambda$ is called the lattice of translations. It is a free abelian group of rank equal to the dimension of $T$.

In this section we recall some definitions and results from [KL2]. Let $G$ be a connected reductive algebraic group over $\mathbb{C}$, with Lie algebra $\mathfrak{g}$. Set $\mathfrak{g}(F)=\mathfrak{g} \otimes_{\mathbb{C}} F$ and 
$\mathfrak{g}(\mathfrak{o})=\mathfrak{g} \otimes \mathfrak{o}$. Denote by Ad the adjoint action of $G_{F}$ on $\mathfrak{g}_{F}$. We often write $K$ for the group $G(\mathfrak{o})$ of $\mathfrak{o}$-points of $G$.

The loop Grassmannian (or affine Grassmannian) is the quotient $X=G(F) / K$. The affine Grassmannian $X$ is an ind-algebraic variety: it is an increasing union (see [KL2])

$$
X_{0} \subset X_{1} \subset X_{2} \subset \cdots \subset X
$$

of finite-dimensional complex projective algebraic varieties. If $H \subset G$ is a connected reductive algebraic subgroup, then the inclusion of $H$ into $G$ induces an injection $H(K) / H(\mathfrak{o}) \hookrightarrow X$ of the loop Grassmannian for $H$ into the loop Grassmannian for $G$.

Each element $\gamma \in \mathfrak{g}(F)$ gives rise to a "vector field" on $X$ whose fixed point set

$$
X_{\gamma}=\left\{x K \in G(F) / K: \operatorname{Ad}\left(x^{-1}\right)(\gamma) \in \mathfrak{g}(\mathfrak{o})\right\}
$$

is called an affine Springer fiber. We say that the element $\gamma$ is compact if $X_{\gamma} \neq \phi$. In [KL2] it is proven that a compact element $\gamma \in \mathfrak{g}(F)$ is regular and semisimple if and only if $X_{\gamma}$ is finite-dimensional, in which case $X_{\gamma}$ is an ind-subvariety of $X$. It is a union of (possibly) infinitely many irreducible components, each of which is a complex projective variety.

\section{CONJECTURE 5.3}

If $\gamma \in \mathfrak{g}(F)$ is compact, regular, and semisimple, then for all $i$ the homology group $H_{i}\left(X_{\gamma} ; \mathbb{C}\right)$ is pure of weight $i$.

In [GKM2] we prove this conjecture for elements $\gamma$ which have "equal valuation."

\subsection{Bruhat decomposition}

Let $T \subset G$ be a maximal torus defined over $\mathbb{C}$ (hence split over $F$ ), with its lattice of translations $\Lambda \subset T(F) \subset G(F)$. The identification $\Lambda \cong T(F) / T(\mathfrak{o})$ induces an embedding $\phi: \Lambda \rightarrow G(F) / G(\mathfrak{o})=X$ of the loop Grassmannian for $T$ into the loop Grassmannian for $G$. Fix a Borel subgroup $B \supset T$, and let $I \subset G(F)$ be the corresponding Iwahori subgroup. Then $G(F)=I \Lambda K$, so $X$ decomposes into $I$ orbits or Bruhat cells:

$$
X=\coprod_{\ell \in \Lambda} I \ell K .
$$

We denote by $C_{\ell}=I \ell x_{0} \subset X$ the orbit (cell) corresponding to $\ell \in \Lambda$.

\subsection{Turning torus}

Each $\lambda \in \mathbb{C}^{\times}$determines an automorphism $\sigma_{\lambda}$ of the field $F=\mathbb{C}((\varepsilon))$ by $\sigma_{\lambda}\left(\varepsilon^{m}\right)=$ $\lambda^{m} \varepsilon^{m}$. We say that $\lambda$ acts on $F$ by "turning the loop," and we refer to the image of 
$\mathbb{C}^{\times} \rightarrow \operatorname{Aut}(F)$ as the "turning torus." The turning torus preserves $\mathfrak{o}=\mathbb{C}[[\varepsilon]]$, and the fixed point set of its action is

$$
\mathfrak{o}^{\mathbb{C}^{\times}}=F^{\mathbb{C}^{\times}}=\mathbb{C} \cdot \varepsilon^{0} .
$$

The resulting action of $\mathbb{C}^{\times}$on $G(F)$ preserves $K=G(\mathfrak{o})$ and induces an action on $X=G(F) / K$ which commutes with the action of $T(\mathbb{C}) \subset I$. On each Bruhat cell $C_{\ell}$, the action of the extended torus

$$
\widetilde{T}(\mathbb{C})=T(\mathbb{C}) \times \mathbb{C}^{\times}
$$

may be described by

$$
(t, \lambda) \cdot g \ell K=\lambda\left(\operatorname{tg} t^{-1}\right) \ell K
$$

\subsection{Affine roots}

Let $\Phi=\Phi(G, T)$ be the root system for $G$, with positive roots $\Phi^{+}$determined by the choice of $B$ and with root space decomposition

$$
\mathfrak{g}(\mathbb{C})=\mathfrak{t}(\mathbb{C}) \oplus \bigoplus_{\alpha \in \Phi} \mathbb{C} Y_{\alpha},
$$

where $Y_{\alpha} \in \mathfrak{g}(\mathbb{C})_{\alpha}$ are root vectors. Let $\widetilde{\Phi}=\{(\alpha, k): \alpha \in \Phi, k \in \mathbb{Z}\}$ be the collection of affine roots. Each affine root $(\alpha, k)$ may be considered to be a character of the extended torus $\widetilde{T}(\mathbb{C})(5.5 .1)$ with $(\alpha, k)(t, \lambda)=\alpha(t) \lambda^{k}$. Then $\widetilde{T}(\mathbb{C})$ acts on the affine root space $\mathbb{C} \varepsilon^{k} Y_{\alpha}$ through this character; that is,

$$
(t, \lambda) \cdot \varepsilon^{k} Y_{\alpha}=\alpha(t) \lambda^{k} \varepsilon^{k} Y_{\alpha} .
$$

Let $C_{0}$ be the fundamental alcove determined by $I$, and let $\widetilde{\Delta}$ be the set of simple affine roots; so

$$
C_{0}=\left\{a \in \chi_{*}(T) \otimes_{\mathbb{Z}} \mathbb{R}: \alpha(a)+k>0 \text { for all }(\alpha, k) \in \widetilde{\Delta}\right\} .
$$

There is a split short exact sequence

$$
1 \rightarrow I_{+} \rightarrow I \rightarrow T(\mathbb{C}) \rightarrow 1
$$

where $I_{+}$denotes the unipotent radical of $I$. Its Lie algebra is given by

$$
\mathfrak{N}(I)=\operatorname{Lie}\left(I_{+}\right)=\prod_{n \in \mathbb{Z}} \varepsilon^{n} T(\mathbb{C}) \oplus \prod_{(\alpha, k)} \mathbb{C} \varepsilon^{k} Y_{\alpha},
$$

where the product is taken over those affine roots $(\alpha, k) \in \widetilde{\Phi}$ such that $\alpha(a)+k>0$ for all $a \in C_{0}$. To fix notation we recall the following standard description of the Bruhat cells. 


\section{LEMMA 5.7}

Let $x_{0}=K \in X$ denote the basepoint. Fix $\ell \in \Lambda$. The exponential map determines a $\widetilde{T}(\mathbb{C})$-equivariant isomorphism between the Bruhat cell $C_{\ell}=I \ell x_{0}$ and the vector space

$$
D_{\ell}=\bigoplus_{(\alpha, k)} \mathbb{C} \varepsilon^{k} Y_{\alpha},
$$

where the sum is over those (finitely many) affine roots $(\alpha, k) \in \widetilde{\Phi}$ such that

$$
\operatorname{val}(\alpha(\ell))+k<0 \quad \text { and } \quad \alpha(a)+k>0 \quad \text { for all } a \in C_{0}
$$

and where $\widetilde{T}(\mathbb{C})$ acts on this vector space according to (5.6.1).

\subsection{Proof}

The subgroup $I_{+}$acts transitively on $C_{\ell}$. The stabilizer $S_{\ell}$ of the point $\ell x_{0}$ is $I_{+} \cap$ $\ell G(\mathfrak{o}) \ell^{-1}$, whose Lie algebra $\mathfrak{s} \ell$ is the sum of the affine root spaces $\mathbb{C}^{k} Y_{\alpha}$ such that $\alpha(a)+k>0$ (for $a \in A$ ) and $\operatorname{Ad}(\ell)\left(\varepsilon^{k} Y_{\alpha}\right) \in \mathfrak{g}(\mathfrak{o})$. The second condition is

$$
\varepsilon^{k+\operatorname{val}(\alpha(\ell))} Y_{\alpha} \in \mathfrak{g}(\mathfrak{o})
$$

or, equivalently, $\operatorname{val}(\alpha(\ell))+k \geq 0$. But (5.7.1) is a $\widetilde{T}(\mathbb{C})$-invariant complement to $\mathfrak{s}_{\ell}$ in $\mathfrak{N}(I)$, so the exponential mapping takes it isomorphically to the Bruhat cell $I \ell x_{0} . \square$

LEMMA 5.9

The fixed point set of the turning torus on $X$ is

$$
X^{\mathbb{C}^{\times}}=G(\mathbb{C}) \Lambda x_{0}
$$

The fixed point set of the torus $T(\mathbb{C})$ on $X$ is

$$
X^{T(\mathbb{C})}=\Lambda x_{0}
$$

\subsection{Proof}

It is easy to see that $G(\mathbb{C}) \Lambda x_{0} \subset X^{\mathbb{C}^{\times}}$. To verify the reverse inclusion, it suffices to show that

$$
X^{\mathbb{C}^{\times}} \cap C_{\ell} \subset G(\mathbb{C}) \Lambda x_{0}
$$

for each Bruhat cell $C_{\ell}$. But the fixed point set of the turning torus on the vector space $D_{\ell}$ consists of those factors in the sum (5.7.1) with $k=0$. So their exponentials are contained in $G(\mathbb{C}) \cap I$. Similarly, the action of $T(\mathbb{C})$ on $D_{\ell}$ has a single fixed point at the origin, so $T(\mathbb{C})$ acts on $C_{\ell}$ with a single fixed point $\ell x_{0}$. 


\subsection{1-dimensional orbits}

Let $X_{1} \subset X$ denote the union of the 0- and 1-dimensional orbits under the action of $T(\mathbb{C})$ on $X$. If $\alpha \in \Phi^{+}$, let $U_{\alpha} \subset G$ be the unique connected $T$-stable 1-dimensional unipotent subgroup whose Lie algebra contains the root space $\mathfrak{g}_{\alpha}$. Then each $\alpha \in \Phi^{+}$ determines a unique reductive connected algebraic subgroup $H_{\alpha} \subset G$ of semisimple rank one which is generated by $T, U_{\alpha}$, and $U_{-\alpha}$. Let $X^{\alpha}=H_{\alpha}(F) / H_{\alpha}(\mathfrak{o})$ be the loop Grassmannian for this subgroup. The inclusion of $H_{\alpha}$ into $G$ induces an injection $X^{\alpha} \hookrightarrow X$.

LEMMA 5.12

The union of the 0 - and 1-dimensional orbits of $T(\mathbb{C})$ on $X$ is

$$
X_{1}=\bigcup_{\alpha \in \Phi^{+}} X^{\alpha}
$$

If $\alpha, \beta \in \Phi^{+}$and $\alpha \neq \beta$, then $X^{\alpha} \cap X^{\beta}=\Lambda$.

\subsection{Proof}

If $\operatorname{dim}(T)=1$, then the result is obvious since both sides coincide with $X$, so we may assume that $\operatorname{dim}(T) \geq 2$. The adjoint action of the torus $T(\mathbb{C})$ on $H_{\alpha}$ factors through an action of the 1-dimensional quotient $T(\mathbb{C}) / \operatorname{ker}(\alpha)$. Hence $X^{\alpha} \subset X_{1}$. To show that $X_{1} \subset \bigcup_{\alpha \in \Phi^{+}} X^{\alpha}$, it suffices to show that

$$
X_{1} \cap C_{\ell} \subset \bigcup_{\alpha \in \Phi^{+}} X^{\alpha} \cap C_{\ell}
$$

for each Bruhat cell $C_{\ell}$. By Lemma 5.7, this amounts to determining the 1dimensional orbits of the action of $T(\mathbb{C})$ on the vector space $D_{\ell}$ of (5.7.1). It follows from (5.6.1) that if $\operatorname{dim}(T) \geq 2$, then these 1-dimensional orbits are precisely the coordinate axes $\mathbb{C} \varepsilon^{k} Y_{\alpha}$. Therefore we may classify the 1-dimensional orbits of $T(\mathbb{C})$ on $D_{\ell}$ by the positive roots, with those orbits corresponding to a fixed $\alpha \in \Phi^{+}$being contained in the subspace

$$
D_{\ell, \alpha}=\bigoplus_{k} \mathbb{C} \varepsilon^{k} Y_{\alpha}=\bigoplus_{k} \varepsilon^{k} \operatorname{Lie}\left(U_{\alpha}\right)(\mathbb{C}) .
$$

Here, the sum is taken over those $k$ such that $\alpha(a)+k>0$ (for all $a \in C_{0}$ ) and $\operatorname{val}(\alpha(\ell))+k<0$. Let $C_{\ell, \alpha} \subset C_{\ell}$ be the corresponding subset of the Bruhat cell. Then $C_{\ell, \alpha}=X^{\alpha} \cap C_{\ell}$, which implies (5.13.1). Finally, if $\alpha \neq \beta$, then $D_{\ell, \alpha} \cap D_{\ell, \beta}=\{0\}$, which proves that $X^{\alpha} \cap X^{\beta}=\Lambda$. 


\section{Affine Springer fibers for SL(2)}

6.1

In this section we fix $G(\mathbb{C})=\operatorname{SL}(2, \mathbb{C})$ and set $X^{\operatorname{SL}(2)}=G(F) / K$ with basepoint $x_{0}=K=G(\mathfrak{o})$. Let $T$ be the torus of diagonal matrices, and let $\alpha$ be the positive root, $\alpha\left(\begin{array}{cc}a & 0 \\ 0 & a^{-1}\end{array}\right)=a^{2}$. Let $\alpha^{\vee}: F^{\times} \rightarrow T(F)$ be the corresponding coroot,

$$
\alpha^{\vee}(b)=\left(\begin{array}{cc}
b & 0 \\
0 & b^{-1}
\end{array}\right)
$$

Then $\Lambda^{\mathrm{SL}(2)}=\left\langle\alpha^{\vee}(\varepsilon)\right\rangle$ is the cyclic group spanned by $\alpha^{\vee}(\varepsilon)$, whose elements we denote by $\ell_{m}=\alpha^{\vee}\left(\varepsilon^{m}\right)$. For any integer $n \leq-1$, set

$$
x_{n}=\left(\begin{array}{cc}
1 & \varepsilon^{n} \\
0 & 1
\end{array}\right) K \in X^{\mathrm{SL}(2)} .
$$

Let $\alpha^{\prime}: \mathfrak{t}(F) \rightarrow F$ be the differential of $\alpha: T(F) \rightarrow F$. The following fact was observed by D. Nadler.

\section{LEMMA 6.2}

The affine Grassmannian $X^{\mathrm{SL}(2)}$ is the disjoint union of countably many $T(F)$-orbits:

$$
X^{\mathrm{SL}(2)}=\coprod_{n \leq 0} T(F) \cdot x_{n} .
$$

The orbit of the point $x_{n}$ has (complex) dimension $|n|$. If $\gamma \in \mathfrak{t}(\mathfrak{o})$ and $v=\operatorname{val}\left(\alpha^{\prime}(\gamma)\right)$, then the affine Springer fiber $X_{\gamma}^{\mathrm{SL}(2)}$ is the union of the $T(F)$-orbits

$$
X_{\gamma}^{\mathrm{SL}(2)}=\bigcup_{n=-v}^{0} T(F) \cdot x_{n}
$$

and it is preserved by the turning torus.

It follows that we may unambiguously denote the affine Springer fiber $X_{\gamma}^{\mathrm{SL}(2)}$ in (6.2.1) by $X_{\leq v}^{\mathrm{SL}(2)}$.

\subsection{Proof}

First observe that every point $x \in X^{\mathrm{SL}(2)}$ has an expression of the form $g K$ with

$$
g=\left(\begin{array}{cc}
\varepsilon^{m} & b_{0} \varepsilon^{n} \\
0 & \varepsilon^{-m}
\end{array}\right),
$$

where either 
(1) $b_{0}=0$ or

(2) $b_{0} \in \mathfrak{o}^{\times}$and $n-m<0$.

In fact, the standard Borel subgroup acts transitively on $X$, so $x=\left(\begin{array}{ll}a & b^{\prime} \\ 0 & a^{-1}\end{array}\right) x_{0}$ for some $a, b^{\prime} \in F$. Set $a=a_{0} \varepsilon^{m}$ with $a_{0} \in \mathfrak{o}^{\times}$, and right multiply by $\alpha^{\vee}\left(a_{0}^{-1}\right) \in K$ to obtain $x=g x_{0}$ with $g=\left(\begin{array}{cc}\varepsilon^{m} & b \\ 0 & \varepsilon^{-m}\end{array}\right)$. If $b=0$ or if $\operatorname{val}(b)<m$, then we are done. Otherwise, set $b=b_{0} \varepsilon^{n}$ with $n \geq m$ and $b_{0} \in \mathfrak{o}^{\times}$. Right multiply by $k=$ $\left(\begin{array}{cc}1 & -b_{0} \varepsilon^{n-m} \\ 0 & 1\end{array}\right) \in K$ to see that $x=g k K \in \Lambda$.

Now let $x=g K$ with $g=\left(\begin{array}{cc}\varepsilon^{m} & b_{0} \varepsilon^{n} \\ 0 & \varepsilon^{-m}\end{array}\right)$. If $b_{0}=0$, then $x=\alpha^{\vee}\left(\varepsilon^{m}\right) x_{0} \in T(F) \cdot x_{0}$. If $b_{0} \in \mathfrak{o}^{\times}$and $m>n$, let $a \in \mathfrak{o}^{\times}$be a square root of $b_{0}$. Set $t=\alpha^{\vee}\left(a \varepsilon^{m}\right) \in T(F)$ and $k=\alpha^{\vee}\left(a^{-1}\right) \in K$. Then $t x_{n-m} k=g$, which proves the first statement. The dimension statement is straightforward. If $\gamma \in \mathfrak{t}(\mathfrak{o})$, then $X_{\gamma}^{\mathrm{SL}(2)}$ is preserved by $T(F)$, so it is a union of $T(F)$-orbits. To verify (6.2.1), suppose $\gamma=\left(\begin{array}{cc}a & 0 \\ 0 & -a\end{array}\right) \in \mathfrak{t}(\mathfrak{o})$. Then $\operatorname{Ad}\left(x_{n}\right) \gamma=\left(\begin{array}{cc}a & -2 a \varepsilon^{n} \\ 0 & -a\end{array}\right)$, which is in $\mathfrak{g}(\mathfrak{o})$ if and only if $\operatorname{val}(a)+n \geq 0$.

Each 1-dimensional orbit $\mathscr{O}$ of $\widetilde{T}(\mathbb{C})$ necessarily contains exactly two fixed points $\ell_{s}, \ell_{t} \in \Lambda$ in its closure, in which case we say that $\mathscr{O}$ "connects" the points $\ell_{s}$ and $\ell_{t}$.

\section{LEMMA 6.4}

For any two lattice points $\ell_{s}, \ell_{t} \in \Lambda$, there exists a unique 1-dimensional orbit $\mathscr{O}_{s t}$ of $\widetilde{T}(\mathbb{C})$ in $X^{\mathrm{SL}(2)}$ which connects them, and this accounts for all the 1-dimensional orbits of $\widetilde{T}(\mathbb{C})$ in $X^{\mathrm{SL}(2)}$. The stabilizer of any point in $\mathscr{O}_{\text {st }}$ is the kernel of the affine root $(\alpha, s+t)$. The orbit $\mathscr{O}_{\text {st }}$ is contained in $X_{\gamma}^{\mathrm{SL}(2)}$ if and only if $\operatorname{val}\left(\alpha^{\prime}(\gamma)\right) \geq|s-t|$.

\subsection{Proof}

By Lemma 6.2, for any $T(\mathfrak{o})$-orbit $\mathscr{T}$ there exists a unique $s \in \mathbb{Z}$ and a unique $n \leq 0$ such that $\ell_{s} x_{n} \in \mathscr{T}$. Set $t=s+n$. We show that

(1) the $\widetilde{T}(\mathbb{C})$-orbit $\mathscr{O}$ of $\ell_{s} x_{n}$ is 1-dimensional,

(2) this is the only 1 -dimensional orbit of $\widetilde{T}(\mathbb{C})$ in $\mathscr{T}$,

(3) $\mathscr{O}$ connects $\ell_{s}$ and $\ell_{t}=\ell_{s}+n \alpha^{\vee}(\varepsilon)$, and

(4) $\quad \widetilde{T}(\mathbb{C})$ acts on $\mathscr{O}$ through the affine root $(\alpha, 2 s+n)$.

First compute the action of $\widetilde{T}(\mathbb{C})$ on the $T(\mathfrak{o})$-orbit of the point $\ell x_{n}$. Let $\alpha^{\vee}(b) \in$ $T(\mathfrak{o})$ with $b=\sum_{i \geq o} b_{i} \varepsilon^{i} \in \mathfrak{o}^{\times}$. Let $a \in \mathbb{C}^{\times}$. Since

$$
\ell_{s} x_{n}=\left(\begin{array}{cc}
\varepsilon^{s} & \varepsilon^{s+n} \\
0 & \varepsilon^{-s}
\end{array}\right)
$$

the action of $\left(\alpha^{\vee}(a), \lambda\right) \in \widetilde{T}(\mathbb{C})$ on the point $\alpha^{\vee}(b) \ell_{s} x_{n}$ is the point

$$
y=\left(\begin{array}{cc}
a(\lambda \cdot b) \lambda^{s} \varepsilon^{s} & a(\lambda \cdot b) \lambda^{s+n} \varepsilon^{s+n} \\
0 & a^{-1}\left(\lambda \cdot b^{-1}\right) \lambda^{-s} \varepsilon^{-s}
\end{array}\right) K .
$$


Right multiply by $\alpha^{\vee}\left(a^{-1}(\lambda \cdot b)^{-1} \lambda^{-s}\right) \in K$ to find that

$$
y=\left(\begin{array}{cc}
\varepsilon^{s} & a^{2}(\lambda \cdot b)^{2} \lambda^{2 s+n} \varepsilon^{s+n} \\
0 & \varepsilon^{-s}
\end{array}\right) K .
$$

As $a, \lambda \in \mathbb{C}^{\times}$vary, this is a 2-dimensional orbit unless $b=b_{0} \in \mathbb{C}^{\times}$, in which case $\lambda \cdot b=b$; hence $\lim _{a \rightarrow 0} y=\ell_{s}$. To find $\lim _{a \rightarrow \infty} y$, choose $\lambda=b=1$, and right multiply by

$$
\left(\begin{array}{cc}
a^{-3} & a^{-1} \varepsilon^{-n}-a^{2} \\
a^{-2} & \varepsilon^{-n}
\end{array}\right) \in K
$$

to find

$$
y=\left(\begin{array}{cc}
a^{-3} \varepsilon^{s}+\varepsilon^{s+n} & a^{-1} \varepsilon^{s-n} \\
a^{-2} \varepsilon^{-s} & \varepsilon^{-s-n}
\end{array}\right) K .
$$

So $y \rightarrow \ell_{s}+n \alpha^{\vee}(\varepsilon)$ as $a \rightarrow \infty$. It follows from (6.5.1) that $\widetilde{T}(\mathbb{C})$ acts on this 1-dimensional orbit through the character $(\alpha, 2 s+n)=(\alpha, s+t)$ (cf. (5.6.1)). This verifies (1)-(4). Finally, by (6.2.1), the orbit $\mathscr{O} \subset X_{\gamma}$ if and only if $|n| \leq \operatorname{val} \alpha^{\prime}(\gamma)$.

\section{Equivariant homology of SL(2) Springer fibers}

\section{1}

As in $\S 6$, let $G=\operatorname{SL}(2)$, let $X=X^{\mathrm{SL}(2)}=G(K) / G(\mathfrak{o})$, and let $\Lambda=\Lambda^{\mathrm{SL}(2)}$. (We use multiplicative notation for the group law in $\Lambda$.) Let $T \subset G$ be the diagonal matrices, with simple root and coroot $\alpha$ and $\alpha^{\vee}$. Then $\alpha^{\vee}(\varepsilon) \in \Lambda$ is a generator (which, by abuse of notation, we denote by $\alpha^{\vee}$ ), so it determines an isomorphism $\mathbb{Z} \cong$ $\Lambda$, written $s \mapsto \ell_{s}$. So $\alpha^{\vee}=\ell_{1}$ and $\alpha^{\vee} \ell_{s}=\ell_{s+1}$. Fix $\gamma \in \mathfrak{t}(\mathfrak{o})$ with corresponding Springer fiber $X_{\gamma}$.

To simplify the notation, for the remainder of $\S 7$ we write $\widetilde{T}$ for $\widetilde{T}(\mathbb{C}), \mathfrak{t}$ for $\mathfrak{t}(\mathbb{C})$, and so on. Let $\mathscr{D}=\mathscr{D}(\mathfrak{t})$ be the algebra of differential operators on $\mathfrak{t}^{*}$, which is identified with $H_{T}^{*}(\mathrm{pt})$ by the Chern-Weil homomorphism. Let $\mathbf{S}(\mathfrak{t})$ be the symmetric algebra of complex-valued polynomial functions on $t^{*}$. The character $\alpha$ corresponds to a differential operator $\partial_{\alpha} \in \mathscr{D}(\mathfrak{t})$. Denote by $\mathbf{S}(\mathfrak{t})\left\{\partial_{\alpha}^{d}\right\}$ the kernel of $\partial_{\alpha}^{d}$. The ChernWeil homomorphism determines an isomorphism of $\mathscr{D}$-modules

$$
H_{*}^{T}(\Lambda) \cong \mathbb{C}[\Lambda] \otimes_{\mathbb{C}} S(\mathfrak{t}) .
$$

PROPOSITION 7.2

Let $v=\operatorname{val}\left(\alpha^{\prime}(\gamma)\right)$. The inclusion $\Lambda \subset X$ induces exact sequences

$$
\begin{aligned}
& H_{*}^{\widetilde{T}}\left(X_{\gamma}, \Lambda\right) \stackrel{\tilde{\beta}}{\longrightarrow} H_{*}^{\widetilde{T}}(\Lambda) \longrightarrow H_{*}^{\widetilde{T}}\left(X_{\gamma}\right) \longrightarrow 0 \\
& \cup \quad U \quad U \\
& H_{*}^{T}\left(X_{\gamma}, \Lambda\right) \stackrel{\beta}{\longrightarrow} H_{*}^{T}(\Lambda) \longrightarrow H_{*}^{T}\left(X_{\gamma}\right) \longrightarrow 0
\end{aligned}
$$


and the image of $\beta$ is the $\mathscr{D}$-submodule

$$
\sum_{d=1}^{v}\left(1-\alpha^{\vee}\right)^{d} \mathbb{C}[\Lambda] \otimes \mathbf{S}(\mathfrak{t})\left\{\partial_{\alpha}^{d}\right\} \subset \mathbb{C}[\Lambda] \otimes \mathbf{S}(\mathfrak{t}) .
$$

\subsection{Proof}

The Springer fiber $X_{\gamma}$ has a paving by affines (see [GKM2]): it is an increasing union of complex projective algebraic varieties $\phi \subset\left(X_{\gamma}\right)_{0} \subset\left(X_{\gamma}\right)_{1} \subset \cdots$ such that each $\left(X_{\gamma}\right)_{j}-\left(X_{\gamma}\right)_{j-1}$ is isomorphic to a single affine space. In fact, the intersection of $X_{\gamma}$ with a single Bruhat cell is either empty or else it is a single affine cell in this paving. It follows that the homology of $\left(X_{\gamma}\right)_{j}$ vanishes in odd degrees. The extended torus $\widetilde{T}=T \times \mathbb{C}^{\times}(\S 5.5)$ preserves each $\left(X_{\gamma}\right)_{j}$, and it acts with finitely many fixed points and finitely many 1-dimensional orbits. So the results of $\S \S 4.1$ and 4.3 may be applied to this action. Since homology commutes with direct limits, we obtain the exact sequences (7.2.1) for both the $\widetilde{T}$-equivariant homology and the $T$-equivariant homology.

The coroot $\alpha^{\vee}$ determines canonical isomorphisms

$$
\mathbf{S}(\widetilde{\mathfrak{t}}) \cong \mathbb{C}[x, t] \quad \text { and } \quad \mathscr{D}(\widetilde{\mathfrak{t}}) \cong \mathbb{C}\left[\partial_{x}, \partial_{t}\right] .
$$

According to Lemma 6.4, for each unordered pair of distinct integers $a, b \in \mathbb{Z}$ such that $|b-a| \leq v=\operatorname{val}\left(\alpha^{\prime}(\gamma)\right)$, there is a unique 1-dimensional orbit $E_{a b} \subset X_{\gamma}$ which connects $\ell_{a}$ and $\ell_{b}$, on which the torus $\widetilde{T}$ acts through the character $\Phi_{a b}$ whose differential $\phi_{a b}: \widetilde{\mathfrak{t}} \rightarrow \mathbb{C}$ corresponds to the differential operator

$$
\partial_{a b}=\partial_{\alpha}+(a+b) \partial_{t}=2 \partial_{x}+(a+b) \partial_{t} .
$$

Let $\mathfrak{m}_{a b}=\operatorname{ker}\left(\phi_{a b}\right)$ denote the Lie algebra of the stabilizer of any point in this orbit, and let $j_{a b}^{*}: \mathbf{S}\left(\mathfrak{m}_{a b}\right) \rightarrow \mathscr{S}(\widetilde{\mathfrak{t}})$ be the resulting inclusion. Then by (4.1.1), the image of $j_{a b}^{*}$ consists of all polynomial functions on $\widetilde{\mathfrak{t}}^{*}$ which are annihilated by the differential operator $\partial_{a b}$. Hence

$$
j_{a b}^{*} \mathbf{S}\left(\mathfrak{m}_{a b}\right)=\{g((a+b) x-2 t): g \in \mathbb{C}[z]\}
$$

consists of polynomial functions of $z=(a+b) x-2 t$. It follows that the image of $\tilde{\beta}$ is the $\operatorname{sum} \sum_{a, b} M_{a b}$ of submodules $M_{a b}$ spanned by elements

$$
\left(\ell_{b}-\ell_{a}\right) \otimes g_{a b}((a+b) x-2 t)
$$

with $|b-a| \leq v$. This is the module $P_{v}$ of $\S 12.6$ (with $2 t$ replaced by $t$ ).

The $T$-equivariant homology of $X_{\gamma}$ may be recovered (see (4.1.1)) from the $\widetilde{T}$ equivariant homology of $X_{\gamma}$ as the kernel of the operator $\partial_{t}$. So the image of $\beta$ is

$$
\operatorname{Im}(\beta)=P_{v} \cap \operatorname{ker}\left(\partial_{t}\right) .
$$

Then Proposition 12.7 identifies $P_{v} \cap \operatorname{ker}\left(\partial_{t}\right)$ with the submodule (7.2.2). 


\section{Groups of semisimple rank one}

For lack of an adequate reference, we include a proof of the following well-known fact.

LEMMA 8.1

Let $H$ be a connected reductive complex linear algebraic group of rank $n$ and of semisimple rank one. Then $H$ is isomorphic to either

(1) $\quad\left(\mathbb{C}^{\times}\right)^{n-1} \times \operatorname{SL}(2, \mathbb{C})$,

(2) $\left(\mathbb{C}^{\times}\right)^{n-1} \times \operatorname{PGL}(2, \mathbb{C})$, or

(3) $\quad\left(\mathbb{C}^{\times}\right)^{n-2} \times \mathrm{GL}(2, \mathbb{C})$.

\subsection{Proof}

Let $\left(L,\{\alpha\}, L^{\vee},\left\{\alpha^{\vee}\right\}\right)$ be the (based) root datum for $H$ where $\langle\cdot, \cdot\rangle: L^{\vee} \times L \rightarrow \mathbb{Z}$ is a dual pairing of rank $n$ lattices, $\alpha \in L, \alpha^{\vee} \in L^{\vee}$, and $\left\langle\alpha^{\vee}, \alpha\right\rangle=2$. If

$$
\left\langle\alpha^{\vee}, \cdot\right\rangle: L \rightarrow \mathbb{Z}
$$

is even-valued, then $(1 / 2) \alpha^{\vee} \in L^{\vee}$, so

$$
L=\left(\alpha^{\vee}\right)^{\perp} \oplus\langle\alpha\rangle \quad \text { while } \quad L^{\vee}=\alpha^{\perp} \oplus\left\langle\frac{1}{2} \alpha^{\vee}\right\rangle,
$$

which corresponds to case (1). (Here $\langle\alpha\rangle$ denotes the cyclic group generated by $\alpha \in L$, and $\left(\alpha^{\vee}\right)^{\perp}$ denotes the kernel of (8.2.1).) Similarly, if $\langle\cdot, \alpha\rangle: L^{\vee} \rightarrow \mathbb{Z}$ is even-valued, then $(1 / 2) \alpha \in L$, so

$$
L=\left(\alpha^{\vee}\right)^{\perp} \oplus\left\langle\frac{1}{2} \alpha\right\rangle \quad \text { while } \quad L^{\vee}=\alpha^{\perp} \oplus\left\langle\alpha^{\vee}\right\rangle,
$$

which corresponds to case (2).

Now suppose that neither of these homomorphisms is even-valued. Let $\left\{e_{1}, e_{2}, \ldots, e_{n-1}\right\}$ be a basis of $L_{0}=\left(\alpha^{\vee}\right)^{\perp}$. Let $L_{1}=\left\{x \in L:\left\langle\alpha^{\vee}, x\right\rangle=1\right\}$. Then $L_{1}-(1 / 2) \alpha$ is a translate of $L_{0}$, so there exist $a_{i} \in\{0,1 / 2\}$ such that $L_{1}-(1 / 2) \alpha=L_{0}+\sum_{i=1}^{n-1} a_{i} e_{i}$ and not all the $a_{i}$ are zero. By relabeling the basis we may assume that $a_{1}=a_{2}=\cdots=a_{r}=1 / 2$ and the remaining coefficients are zero. Let $v=e_{1}+e_{2}+\cdots+e_{r}$. Then $\left\{v, e_{2}, \ldots, e_{r}\right\}$ is also a basis for $L_{0}$, and $(1 / 2) \alpha+(1 / 2) v \in L$. It follows that

$$
L=Y \oplus\left\langle e_{2}, e_{3}, \ldots, e_{n-1}\right\rangle,
$$

where

$$
Y=\left\{m \alpha+m^{\prime} v: m, m^{\prime} \in \mathbb{Z} \text { or } m, m^{\prime} \in \mathbb{Z}+\frac{1}{2}\right\} .
$$

Then this corresponds to case (3), with $L^{\vee}=\left\langle e_{2}, e_{3}, \ldots, e_{n-1}\right\rangle^{\perp} \oplus Y^{\perp}$. 


\section{3}

Let $H$ be a connected reductive complex linear algebraic group of semisimple rank one. Fix a Borel pair $B \subset T \subset H$ with resulting positive root $\alpha$. Let $X^{H}$ be the affine Grassmannian for $H$, and let $\Lambda^{H} \subset X^{H}$ be the lattice of translations for $T$. Consider the abstract $\operatorname{SL}(2, \mathbb{C})$ which corresponds to the simple root $\alpha$. The canonical mapping $\mathrm{SL}(2) \rightarrow H$ induces an inclusion $\phi_{\alpha}: X^{\mathrm{SL}(2)} \subset X^{H}$ with $\phi_{\alpha}\left(\Lambda^{\mathrm{SL}(2)}\right)=$ $\left\langle\alpha^{\vee}(\varepsilon)\right\rangle \subset \Lambda^{H}$ (the infinite cyclic subgroup generated by $\alpha^{\vee}(\varepsilon)$ ). To simplify the notation, identify the coroot $\alpha^{\vee}$ with its image $\alpha^{\vee}(\varepsilon)$ in $\Lambda^{H}$.

\section{LEMMA 8.4}

The following statements hold.

(1) The affine Grassmannian for $H$ is the disjoint union

$$
X^{H}=\coprod_{\ell \in \Lambda^{H} /\left\langle\alpha^{\vee}\right\rangle} \ell \phi_{\alpha}\left(X^{\mathrm{SL}(2)}\right)
$$

(2) The $T^{H}(\mathfrak{o})$-orbits on $X^{H}$ coincide with the $T^{\mathrm{SL}(2)}(\mathfrak{o})$-orbits on $X^{H}$ (which are in turn translates, by elements of $\Lambda^{H}$, of $T^{\mathrm{SL}(2)}(\mathfrak{o})$-orbits on $\left.X^{\mathrm{SL}(2)}\right)$.

(3) Let $\gamma \in \mathfrak{t}^{H}(\mathfrak{o})$ be a regular element. Then the affine Springer fiber $X_{\gamma}^{H}$ is the disjoint union

$$
X_{\gamma}^{H}=\coprod_{\ell \in \Lambda^{H} /\left\langle\alpha^{\vee}\right\rangle} \ell \phi_{\alpha}\left(X_{\leq v}^{\mathrm{SL}(2)}\right)
$$

where $v=\operatorname{val}\left(\alpha^{\prime}(\gamma)\right)(c f$. equation (6.2.1)).

\subsection{Proof}

For part (1), use Lemma 8.1 to reduce to the case $H=\mathrm{GL}(2)$ or the case $H=$ PGL(2). If $H=\mathrm{GL}(2)$, then the exact sequences

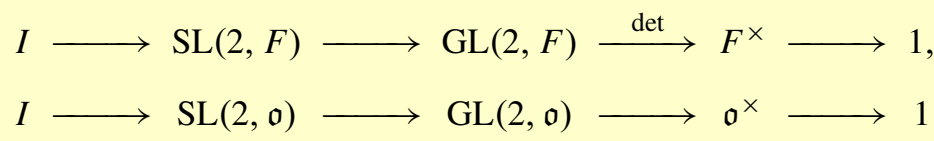

together with the isomorphism val : $F^{\times} / \mathfrak{o}^{\times} \cong \mathbb{Z}$ give rise to a diagram

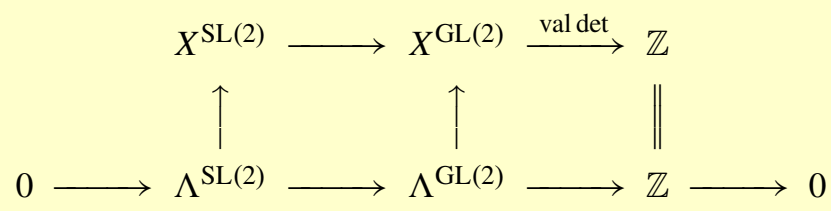

from which the result follows. Now suppose $H=$ PGL(2). If $g \in H(F)$, let $\tau(g)=$ valdet $g \bmod 2$. If $\tau(g)=0$, then $\operatorname{det} g$ has a square root in $F$ and 
$g / \sqrt{\operatorname{det} g} \in \operatorname{SL}(2, F)$. So the exact sequences

$$
\begin{aligned}
& \{ \pm I\} \longrightarrow \operatorname{SL}(2, F) \longrightarrow \operatorname{PGL}(2, F) \stackrel{\tau}{\longrightarrow} \mathbb{Z} /(2), \\
& \{ \pm I\} \longrightarrow \operatorname{SL}(2, \mathfrak{o}) \longrightarrow \operatorname{PGL}(2, \mathfrak{o}) \longrightarrow 1
\end{aligned}
$$

give rise to a diagram

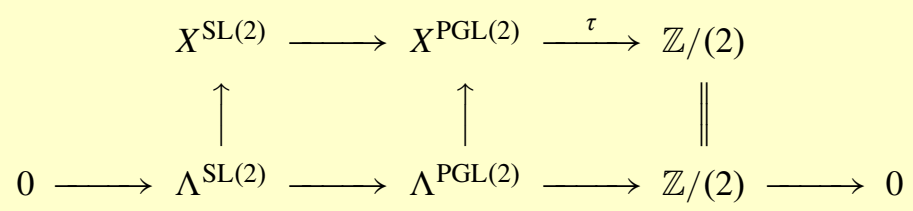

from which the result follows.

For part (2) we may assume $H=\mathrm{GL}(2)$ or PGL(2). Let $g K \in X^{H}$, and let $t=\left(\begin{array}{ll}a & 0 \\ 0 & d\end{array}\right) \in T^{H}(\mathfrak{o})$. Then $a d^{-1}$ has a square root $\lambda \in \mathfrak{o}$. Set $t^{\prime}=\alpha^{\vee}(\lambda) \in T^{\operatorname{SL}(2)}(\mathfrak{o})$. By matrix multiplication, $\operatorname{tg} t^{-1}=t^{\prime} g\left(t^{\prime}\right)^{-1}$; hence $\operatorname{tg} K^{H}=t^{\prime} g K^{H}$.

Now consider part (3). It is easy to verify for $H=\mathrm{GL}(2)$, for $H=\operatorname{PGL}(2)$, and for $n \leq-1$ that $x_{n} \in X_{\gamma}^{H}$ if and only if $|n| \leq v=\operatorname{val}\left(\alpha^{\prime}(\gamma)\right)$, where $x_{n}=\left(\begin{array}{cc}1 & \varepsilon^{n} \\ 0 & 1\end{array}\right) K$. Since $X_{\gamma}^{H}$ is a union of $T^{\mathrm{SL}(2)}(\mathfrak{o})$-orbits, it follows that

$$
X_{\gamma}^{H} \cap \phi_{\alpha}\left(X^{\mathrm{SL}(2)}\right)=\phi_{\alpha}\left(X_{\leq v}^{\mathrm{SL}(2)}\right) .
$$

By Lemma 8.1, equation (8.5.1) holds for any $H$ of semisimple rank one. It follows that the union (8.4.1) is contained in $X_{\gamma}^{H}$. Conversely, if $\mathscr{O}$ is a $T^{H}(\mathfrak{o})$-orbit in $X_{\gamma}^{H}$, then there exists $\ell \in \Lambda^{H}$ such that $\ell \phi_{\alpha}\left(x_{n}\right) \in \mathscr{O}$, which holds if and only if $\phi_{\alpha}\left(x_{n}\right) \in$ $X_{\gamma}^{H}$ or $x_{n} \in X_{\leq v}^{\mathrm{SL}(2)}$. So

$$
X_{\gamma}^{H} \subset \bigcup_{\ell \in \Lambda^{H}} \ell \phi_{\alpha}\left(X_{\leq v}^{\mathrm{SL}(2)}\right) .
$$

The normalizer of $\phi_{\alpha}\left(X_{\leq v}^{\mathrm{SL}(2)}\right)$ in $\Lambda^{H}$ is the sublattice $\left\langle\alpha^{\vee}\right\rangle=\phi_{\alpha}\left(\Lambda^{\mathrm{SL}(2)}\right)$, so the union (8.4.1) is disjoint.

\section{Equivariant homology of affine Springer fibers}

9.1

As in $\$ 5$, we consider a connected reductive linear algebraic group $G$ defined over $\mathbb{C}$ and a Borel pair $T \subset B \subset G$ with a resulting system of positive roots $\Phi^{+}$. Fix $\gamma \in \mathfrak{t}(\mathfrak{o})$, and let $X_{\gamma}$ be the resulting affine Springer fiber. It contains the lattice $\Lambda=\Lambda^{G}$. We use multiplicative notation for the group operation in $\Lambda$. For notational simplicity, in this section we denote by $\mathfrak{t}, \mathfrak{t}^{*}$, and so on, the complex vector spaces $\mathfrak{t}(\mathbb{C}), \mathfrak{t}^{*}(\mathbb{C})$, and so on. Let $\alpha \in \Phi^{+}$, and let $\alpha^{\vee}: F^{\times} \rightarrow T(F)$ be the corresponding 
coroot; it determines a lattice element that we also denote by $\alpha^{\vee} \in \Lambda$. Moreover, $\alpha^{\vee} \in \mathfrak{t}=\mathfrak{t}^{* *}$ corresponds to a (degree 1) monomial

$$
x_{\alpha} \vee \in \mathbf{S}(\mathfrak{t}),
$$

while $\alpha \in \mathfrak{t}^{*}$ corresponds to a (degree 1 ) differential operator

$$
\partial_{\alpha} \in \mathscr{D}(\mathfrak{t})
$$

such that $\partial_{\alpha}\left(x_{\alpha^{\vee}}\right)=2$. Let $\mathbf{S}(\mathfrak{t})\left\{\partial_{\alpha}^{d}\right\}$ be the submodule of polynomial functions that are annihilated by the differential operator $\partial_{\alpha}^{d}$. Define the following submodule of $H_{*}^{T(\mathbb{C})}(\Lambda)=\mathbb{C}[\Lambda] \otimes \mathbf{S}(\mathfrak{t}):$

$$
L_{\alpha, \gamma}=\sum_{d=1}^{\operatorname{val}\left(\alpha^{\prime}(\gamma)\right)}\left(1-\alpha^{\vee}\right)^{d} \mathbb{C}[\Lambda] \otimes \mathbf{S}(\mathfrak{t})\left\{\partial_{\alpha}^{d}\right\} .
$$

THEOREM 9.2

Suppose that the homology $H_{*}\left(X_{\gamma} ; \mathbb{C}\right)$ is pure. Then the inclusion $\Lambda \subset X_{\gamma}$ induces an exact sequence

$$
0 \longrightarrow \sum_{\alpha \in \Phi^{+}} L_{\alpha, \gamma} \longrightarrow \mathbb{C}[\Lambda] \otimes \mathbf{S}(\mathfrak{t}) \longrightarrow H_{*}^{T(\mathbb{C})}\left(X_{\gamma}\right) \longrightarrow 0 .
$$

\subsection{Proof}

Since the homology of $X_{\gamma}$ is equivariantly formal, the exact sequence (4.3.1) becomes

$$
H_{*}^{T(\mathbb{C})}\left(\left(X_{\gamma}\right)_{1}, \Lambda\right) \stackrel{\beta}{\longrightarrow} H_{*}^{T(\mathbb{C})}(\Lambda) \longrightarrow H_{*}^{T(\mathbb{C})}\left(X_{\gamma}\right) \longrightarrow 0,
$$

where $\left(X_{\gamma}\right)_{1}$ denotes the closure of the set of 1-dimensional $T(\mathbb{C})$-orbits in $X_{\gamma}$. By Lemma 5.12, $X_{\gamma}^{\alpha} \cap X_{\gamma}^{\beta}=\Lambda$ if $\alpha \neq \beta$, and

$$
\left(X_{\gamma}\right)_{1}=\bigcup_{\alpha \in \Phi^{+}} X_{\gamma}^{\alpha}
$$

where $X_{\gamma}^{\alpha}$ is the affine Springer fiber corresponding to $\gamma$ in the loop Grassmannian $X^{\alpha}$ for the group $H^{\alpha}$ of semisimple rank one which is determined by $\alpha$. So the image of $\beta$ is the sum over positive roots of the image of the corresponding mapping in the semisimple rank one case. Thus it suffices to consider the case in which the group $G$ has semisimple rank one, which we now assume.

Let $A=\operatorname{ker}(\alpha)^{0} \subset T$, and let $T_{1} \subset T$ be the 1-dimensional subtorus corresponding to the coroot $\alpha^{\vee}$. The canonical decomposition $\mathfrak{t}=\mathfrak{t}_{1} \oplus \mathfrak{a}$ determines an isomorphism $H_{*}^{T(\mathbb{C})}(\mathrm{pt}) \cong H^{T_{1}(\mathbb{C})}(\mathrm{pt}) \otimes H_{*}^{A(\mathbb{C})}(\mathrm{pt})$; that is, $\mathbf{S}(\mathfrak{t}) \cong \mathbf{S}\left(\mathfrak{t}_{1}\right) \otimes \mathbf{S}(\mathfrak{a})$ and

$$
\mathbf{S}(\mathfrak{t})\left\{\partial_{\alpha}^{d}\right\} \cong \mathbf{S}\left(\mathfrak{t}_{1}\right)\left\{\partial_{\alpha}^{d}\right\} \otimes \mathbf{S}(\mathfrak{a}) \text {. }
$$


So the exact sequence $(9.3 .1)$ for $T(\mathbb{C})$-equivariant homology is obtained from the same exact sequence for $T_{1}(\mathbb{C})$-equivariant homology,

$$
H_{*}^{T_{1}(\mathbb{C})}\left(X_{\gamma}, \Lambda\right) \stackrel{\beta_{1}}{\longrightarrow} H_{*}^{T_{1}(\mathbb{C})}(\Lambda) \longrightarrow H_{*}^{T_{1}(\mathbb{C})}\left(X_{\gamma}\right) \longrightarrow 0
$$

by tensoring with $\mathbf{S}(\mathfrak{a})$. By Lemma 8.4, the homomorphism $\beta_{1}$ decomposes as a direct sum of homomorphisms:

$$
\bigoplus_{\ell \in \Lambda^{H} /\left\langle\alpha^{\vee}\right\rangle} \ell_{*} \phi_{\alpha *} H_{*}^{T_{1}(\mathbb{C})}\left(X_{\leq v}^{\mathrm{SL}(2)}, \Lambda^{\mathrm{SL}(2)}\right) \longrightarrow \bigoplus_{\ell \in \Lambda^{H} /\left\langle\alpha^{\vee}\right\rangle} \ell_{*} \phi_{\alpha *} H_{*}^{T_{1}(\mathbb{C})}\left(\Lambda^{\mathrm{SL}(2)}\right)
$$

(where $\phi_{\alpha *}$ and $\ell_{*}$ denote the homomorphisms on homology which are induced by $\phi_{\alpha}$ and by translation by $\ell \in \Lambda^{H}$, resp.). So by Proposition 7.2, the image of $\beta_{1}$ is the sum

$$
\begin{aligned}
\sum_{\ell \in \Lambda^{H} / \Lambda^{\mathrm{SL}(2)}} \ell_{*} \phi_{\alpha *} \sum_{d=1}^{v}\left(\alpha^{\vee}-1\right)^{d} \mathbb{C}\left[\Lambda^{\mathrm{SL}(2)}\right] \otimes & \mathbf{S}\left(\mathfrak{t}_{1}\right)\left\{\partial_{\alpha}^{d}\right\} \\
& =\sum_{d=1}^{v}\left(\alpha^{\vee}-1\right)^{d} \mathbb{C}[\Lambda] \otimes \mathbf{S}\left(\mathfrak{t}_{1}\right)\left\{\partial_{\alpha}^{d}\right\} .
\end{aligned}
$$

Since the image of $\beta$ is obtained by tensoring with $\mathbf{S}(\mathfrak{a})$, we obtain

$$
\operatorname{Im}(\beta)=\sum_{d=1}^{v}\left(\alpha^{\vee}-1\right)^{d} \mathbb{C}[\Lambda] \otimes \mathbf{S}(\mathfrak{t})\left\{\partial_{\alpha}^{d}\right\} .
$$

In fact, in the semisimple rank one case, as a module over $\mathbf{S}(\mathfrak{a})$, the vector space of relations $L_{\alpha, \gamma}$ has a basis consisting of the collection of elements

$$
f_{\ell, d, \alpha}=\left(1-\alpha^{\vee}\right)^{d} \ell \otimes x_{\alpha}^{d-1} \in \mathbb{C}[\Lambda] \otimes \mathbf{S}(\mathfrak{t})
$$

with $\ell \in \Lambda$ and $1 \leq d \leq \operatorname{val}\left(\alpha^{\prime}(\gamma)\right)$.

9.4

Let Aut denote the automorphism group of the based root datum for $G$. Let $W=$ $W(G, T)$ be the Weyl group, and let $\widetilde{W}=\Lambda \rtimes W$ be the extended affine Weyl group. Then the group

$$
\widetilde{W} \rtimes \mathbf{A u t}
$$

acts on $T$ and on $\Lambda$. It acts through $W \rtimes$ Aut on $T(\mathbb{C})$ and on the root system $\Phi(G, T)$. Consider the diagonal action (from the left) of the group (9.4.1) on the equivariant homology

$$
H_{*}^{T(\mathbb{C})}(\Lambda) \cong \mathbb{C}[\Lambda] \otimes_{\mathbb{C}} \mathbf{S}(\mathfrak{t}) .
$$


It preserves the kernel

$$
L_{\infty}=\sum_{\alpha \in \Phi^{+}} \sum_{d=1}^{\infty}\left(1-\alpha^{\vee}\right)^{d} \mathbb{C}[\Lambda] \otimes \mathbf{S}(\mathfrak{t})\left\{\partial_{\alpha}^{d}\right\}
$$

of the surjection $H_{*}^{T(\mathbb{C})}(\Lambda) \rightarrow H_{*}^{T(\mathbb{C})}(X)$, so it passes to an action on the equivariant homology of $X$, which we refer to as the "left" action. If $\gamma \in \mathfrak{t}(\mathfrak{o})$, then the subgroup

$$
(\widetilde{W} \rtimes \mathbf{A u t})_{\gamma}=\left\{\tau \in \widetilde{W} \rtimes \operatorname{Aut}: \operatorname{val}(\tau \alpha(\gamma))=\operatorname{val}(\alpha(\gamma)) \text { for all } \alpha \in \Phi^{+}\right\}
$$

preserves the module of relations

$$
\sum_{\alpha \in \Phi^{+}} L_{\alpha, \gamma} \subset H_{*}^{T(\mathbb{C})}(\Lambda)
$$

Consequently, if the homology $H_{*}\left(X_{\gamma}\right)$ is pure, the group $(\widetilde{W} \rtimes \mathbf{A u t})_{\gamma}$ acts (from the left) on the equivariant homology $H^{T(\mathbb{C})}\left(X_{\gamma}\right)$ by $\mathscr{D}(\mathfrak{t})$ module homomorphisms. By (4.1.1), the ordinary homology is the submodule $H_{*}\left(X_{\gamma}\right)=H_{*}^{T(\mathbb{C})}\left(X_{\gamma}\right)\{\mathscr{I}\}$ which is killed by the augmentation ideal $\mathscr{I}$, so we obtain an action of $(\widetilde{W} \rtimes \text { Aut })_{\gamma}$ on the ordinary homology of the affine Springer fiber.

We remark that in many cases (including $G=\mathrm{SL}(n), \operatorname{Sp}(n)$, or $O(n)$ ) it is possible to construct a (continuous) action of the group (9.4.1) on $X$ which is $T(\mathbb{C})$ covariant, meaning that $\tau(t \cdot x)=\tau(t) \cdot \tau(x)$ for $t \in T(\mathbb{C}), x \in X$, and $\tau \in \widetilde{W} \rtimes$ Aut. The induced action on $H_{*}^{T(\mathbb{C})}(X)$ then agrees with the left action. However, even in these cases, the topological action of the subgroup (9.4.2) does not necessarily preserve the Springer fiber $X_{\gamma}$.

\section{Endoscopic groups}

10.1

In this section we assume, for simplicity only, that $G$ is adjoint. Fix endoscopic data $(H, s)$ for $G$. This means that $H$ is a connected reductive complex algebraic group, that $s \in \widehat{T} \subset \widehat{G}$, and that $\widehat{H}=\widehat{G}_{s}$ is the centralizer of $s$ in $\widehat{G}$. Then $\widehat{H}$ is connected since $\widehat{G}$ is simply connected (see [Hu, §2.11]). We assume, moreover, that Borel pairs $T \subset B \subset G$ and $T_{H} \subset B_{H} \subset H$ (defined over $\mathbb{C}$ ) have been chosen, giving rise to a canonical isomorphism

$$
T_{H} \cong T .
$$

Then $\widehat{T}=T_{\widehat{H}}$ is also a maximal torus in $\widehat{H}$. The set of coroots of $T$ in $H$ is

$$
\Phi^{\vee}\left(H, T_{H}\right)=\left\{\alpha^{\vee} \in \Phi^{\vee}(G, T): s\left(\alpha^{\vee}\right)=1\right\} .
$$


Let $\mathscr{D}=\mathscr{D}(\mathfrak{t}) \cong \mathbf{S}\left(\mathfrak{t}^{*}\right)$ be the graded algebra of differential operators on $\mathfrak{t}^{*}$. Each $\alpha \in \Phi(G, T)$ determines a differential operator $\partial_{\alpha} \in \mathscr{D}$ of degree 1 . Define the homological transfer factor

$$
\Delta=\prod_{\alpha \in \Phi^{+}(G)-\Phi^{+}(H)} \partial_{\alpha}^{\mathrm{val} \alpha^{\prime}(\gamma)} \in \mathscr{D}=H_{T(\mathbb{C})}^{*}(\mathrm{pt}) .
$$

It is a homogeneous element of some degree, $r=\sum_{\alpha \in \Phi^{+}(G)-\Phi^{+}(H)} \operatorname{val} \alpha^{\prime}(\gamma)$. The action of $\Delta$ is a surjection of graded $\mathscr{D}$-modules

$$
\mathbf{S}(\mathfrak{t}) \stackrel{\Delta \cdot}{\longrightarrow} \mathbf{S}(\mathfrak{t})[-r] \longrightarrow 0
$$

(where $A[-r]$ denotes the shift in grading and $A[-r]_{k}=A_{k-r}$ for any graded vector space $\left.A=\bigoplus_{k} A_{k}\right)$.

Fix $\gamma \in \mathfrak{t}(\mathfrak{o})$ regular and semisimple, and let $\gamma_{H} \in \mathfrak{t}_{H}=\operatorname{Lie}\left(T_{H}\right)$ be the element that corresponds to $\gamma$ under the isomorphism (10.1.1). Then $\gamma_{H}$ acts as a "vector field" on the affine Grassmannian $X^{H}=H(F) / H(\mathfrak{o})$, and we denote its fixed point set by $X_{\gamma_{H}}^{H}$.

Let Aut ${ }^{G, H}$ be the subgroup of the automorphism group of the based root datum for $G$ which preserves the roots of $H$. Let $W^{H}$ be the Weyl group for $H$, set $\widetilde{W}^{H}=$ $\Lambda \rtimes W^{H}$ and $\widetilde{\mathscr{W}}^{G, H}=\widetilde{W}^{H} \rtimes \mathbf{A u t}^{G, H}$, and define a subgroup of $\widetilde{\mathscr{W}}^{G, H}$ by

$$
\widetilde{\mathscr{W}}_{\gamma}^{G, H}=\left\{\tau \in \widetilde{\mathscr{W}}^{G, H}: \operatorname{val}\left(\tau \alpha^{\prime}(\gamma)\right)=\operatorname{val}\left(\alpha^{\prime}(\gamma)\right) \text { for all } \alpha \in \Phi^{+}(G, T)\right\} .
$$

Note that the square of $\Delta$ is invariant under $\widetilde{\mathscr{W}}_{\gamma}^{G, H}$ and hence that there exists a sign character

$$
\eta: \widetilde{\mathscr{W}}_{\gamma}^{G, H} \rightarrow\{ \pm 1\}
$$

such that $\tau(\Delta)=\eta(\tau) \cdot \Delta$ for all $\tau \in \widetilde{\mathscr{W}}_{\gamma}^{G, H}$.

Let $J \subset \mathbb{C}[\Lambda]$ be the multiplicative subset that is generated by the elements $1-\alpha^{\vee}$ for $\alpha^{\vee} \in \Phi^{\vee}(G, T)-\Phi^{\vee}\left(H, T_{H}\right)$. For any $\mathbb{C}[\Lambda]$-module $M$, let $M_{J}=J^{-1} M$ denote the $\mathbb{C}[\Lambda]_{J}=J^{-1} \mathbb{C}[\Lambda]$ module that is obtained by inverting the elements of $J$. The localization of the module $\mathbb{C}[\Lambda] \otimes \mathbf{S}(\mathfrak{t})$ is $\mathbb{C}[\Lambda]_{J} \otimes \mathbf{S}(\mathfrak{t})$.

THEOREM 10.2

Suppose that the homology of $X_{\gamma}$ and of $X_{\gamma_{H}}^{H}$ is pure. Then the surjection

$$
1 \otimes \Delta: \mathbb{C}[\Lambda] \otimes_{\mathbb{C}} \mathbf{S}(\mathfrak{t}) \rightarrow \mathbb{C}[\Lambda] \otimes_{\mathbb{C}} \mathbf{S}(\mathfrak{t})[-r]
$$

induces a homomorphism of $\mathscr{D}$-modules

$$
\Psi: H_{*}^{T(\mathbb{C})}\left(X_{\gamma}\right) \rightarrow H_{*}^{T(\mathbb{C})}\left(X_{\gamma_{H}}^{H}\right)[-2 r]
$$


which becomes an isomorphism

$$
\Psi_{J}: H_{*}^{T(\mathbb{C})}\left(X_{\gamma}\right)_{J} \rightarrow H_{*}^{T(\mathbb{C})}\left(X_{\gamma_{H}}^{H}\right)_{J}[-2 r]
$$

after localizing with respect to J. The homomorphisms $\Psi$ and $\Psi_{J}$ are not quite $\widetilde{\mathscr{W}}_{\gamma}^{G, H}$-equivariant; rather, they transform under $\widetilde{\mathscr{W}}_{\gamma}^{G, H}$ by the sign character $\eta$ defined above.

Assuming that the homology of $X_{\gamma}$ is pure, by Lemma 2.2 and equation (4.1.1), the ordinary homology of $X_{\gamma}$ is given by the $\mathscr{D}$-submodule of the equivariant homology

$$
H_{*}\left(X_{\gamma} ; \mathbb{C}\right) \cong H_{*}^{T(\mathbb{C})}\left(X_{\gamma} ; \mathbb{C}\right)\{\mathscr{I}\}
$$

which is annihilated by the augmentation ideal $\mathscr{I} \subset \mathscr{D}$. We conclude the following.

COROLLARY 10.3

The action of $1 \otimes \Delta$ on the equivariant homology induces a homomorphism $H_{*}\left(X_{\gamma}\right) \rightarrow H_{*}\left(X_{\gamma_{H}}^{H}\right)[-2 r]$ and an isomorphism

$$
H_{*}\left(X_{\gamma} ; \mathbb{C}\right)_{J} \cong H_{*}\left(X_{\gamma_{H}}^{H} ; \mathbb{C}\right)_{J}[-2 r] .
$$

These maps again transform under $\widetilde{\mathscr{W}}_{\gamma}^{G, H}$ by the sign character $\eta$.

Although the homomorphism (10.2.2) may be described relatively easily in equivariant homology, the resulting isomorphism (10.3.1) in ordinary homology is much more complicated.

\subsection{Proof of Theorem 10.2}

The mapping $\Psi$ is well defined because the mapping (10.2.1) kills the submodule

$$
L_{\alpha, \gamma}=\sum_{d=1}^{\operatorname{val}\left(\alpha^{\prime}(\gamma)\right)}\left(1-\alpha^{\vee}\right)^{d} \mathbb{C}[\Lambda] \otimes \mathbf{S}(\mathfrak{t})\left\{\partial_{\alpha}^{d}\right\}
$$

whenever $\alpha \in \Phi^{+}(G)-\Phi^{+}(H)$. If $w \in \widetilde{W} \rtimes$ Aut and if $f \in \mathbf{S}(\mathfrak{t})$, then $w(\Delta f)=$ $w(\Delta) w(f)$. If $w$ lies in the subgroup $\widetilde{\mathscr{W}}_{\gamma}^{G, H}$, then $w(\Delta)=\eta(w) \cdot \Delta$. Therefore the maps $1 \otimes \Delta$ and $\Psi$ transform under $\widetilde{\mathscr{W}}_{\gamma}^{G, H}$ by the sign character $\eta$.

Now let us check that $\Psi_{J}$ is an isomorphism. The surjection

$$
(1 \otimes \Delta)_{J}: \mathbb{C}[\Lambda]_{J} \otimes \mathbf{S}(\mathfrak{t}) \rightarrow \mathbb{C}[\Lambda]_{J} \otimes \mathbf{S}(\mathfrak{t})[-r]
$$

has kernel

$$
\operatorname{ker}(1 \otimes \Delta)_{J}=\mathbb{C}[\Lambda]_{J} \otimes \mathbf{S}(\mathfrak{t})\{\Delta\}
$$


and it restricts to a mapping $h_{J}$ in the following diagram:

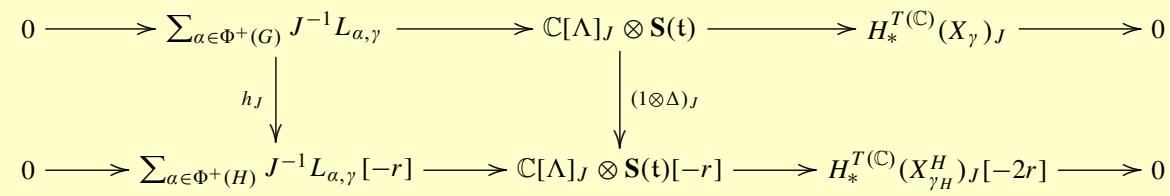

It suffices (by the snake lemma) to show that $h_{J}$ is surjective and that

$$
\operatorname{ker}(1 \otimes \Delta)_{J} \subset \sum_{\alpha \in \Phi^{+}(G)} J^{-1} L_{\alpha, \gamma}
$$

If $\alpha \in \Phi^{+}(G)-\Phi^{+}(H)$ (i.e., if $s\left(\alpha^{\vee}\right) \neq 1$ ), then $\left(1-\alpha^{\vee}\right.$ ) becomes invertible in $\mathbb{C}[\Lambda]_{J}$. If $a \leq b$, then $\mathbf{S}(\mathfrak{t})\left\{\partial_{\alpha}^{a}\right\} \subset \mathbf{S}(\mathfrak{t})\left\{\partial_{\alpha}^{b}\right\}$. Therefore the localization of the submodule $L_{\alpha, \gamma}$ is

$$
J^{-1} L_{\alpha, \gamma}=\mathbb{C}[\Lambda]_{J} \otimes \mathbf{S}(\mathfrak{t})\left\{\partial_{\alpha}^{\operatorname{val}\left(\alpha^{\prime}(\gamma)\right)}\right\} .
$$

By Lemma 3.2, the sum

$$
\sum_{\substack{\alpha \in \Phi^{+}(G) \\ s\left(\alpha^{\vee}\right) \neq 1}} \mathbf{S}(\mathfrak{t})\left\{\partial_{\alpha}^{\operatorname{val}\left(\alpha^{\prime}(\gamma)\right)}\right\}=\mathbf{S}(\mathfrak{t})\{\Delta\}
$$

is killed by $\Delta$. Therefore

$$
\operatorname{ker}(1 \otimes \Delta)_{J}=\sum_{\substack{\alpha \in \Phi^{+}(G) \\ s\left(\alpha^{\vee}\right) \neq 1}} J^{-1} L_{\alpha, \gamma}
$$

which proves (10.4.1). On the other hand, if $\alpha \in \Phi^{+}(G)$ and $s\left(\alpha^{\vee}\right)=1$, then

$$
J^{-1} L_{\alpha, \gamma}=\sum_{d=1}^{\operatorname{val}\left(\alpha^{\prime}(\gamma)\right)}\left(1-\alpha^{\vee}\right)^{d} \mathbb{C}[\Lambda]_{J} \otimes \mathbf{S}(\mathfrak{t})\left\{\partial_{\alpha}^{d}\right\} .
$$

In this case (by Lemma 3.2) the action of $\Delta$ is a surjection

$$
\mathbf{S}(\mathfrak{t})\left\{\partial_{\alpha}^{d}\right\} \rightarrow \mathbf{S}(\mathfrak{t})\left\{\partial_{\alpha}^{d}\right\}[-r],
$$

which implies that $h_{J}$ is surjective.

\subsection{Remark}

We did not use the full strength of the endoscopic hypothesis on $H$, and in fact, Theorem 10.2 remains valid whenever $H$ is a connected reductive group for which there exists a Borel pair $T_{H} \subset B_{H}$ and an isomorphism $T \cong T_{H}$ such that the set of positive roots $\Phi^{+}\left(H, T_{H}\right)$ is a subset of the positive roots $\Phi^{+}(G, T)$. 


\section{The quotient under $\Lambda$}

11.1

As in $\S 10$, fix a Borel pair $T \subset B \subset G$ with resulting positive roots $\Phi^{+}$. Fix regular $\gamma \in \mathfrak{t}(\mathfrak{o})$ with affine Springer fiber $X_{\gamma}$ and its lattice of translations $\Lambda$. The quotient $\Lambda \backslash X_{\gamma}$ admits the structure of a complex projective algebraic variety (see [KL2]). Let $(H, s)$ be endoscopic data with $s \in \widehat{T}$, and assume that $s$ has finite order. View $s$ as a character $s: \Lambda \rightarrow \mathrm{GL}_{1}(\mathbb{C})$, and let $\mathbb{C}_{s}$ be the 1-dimensional $\mathbb{C}[\Lambda]$-module defined by $s$. Then $s$ determines 1-dimensional local systems

$$
\mathscr{L}_{s}=X_{\gamma} \times{ }_{\Lambda} \mathbb{C}_{s} \quad \text { and } \quad \mathscr{M}_{s}=X_{\gamma_{H}}^{H} \times{ }_{\Lambda} \mathbb{C}_{s}
$$

on $\Lambda \backslash X_{\gamma}$ and $\Lambda \backslash X_{\gamma_{H}}^{H}$, respectively. Let $r=\operatorname{deg}(\Delta)$ as in (10.1.2).

PROPOSITION 11.2

Suppose that $H_{*}\left(X_{\gamma} ; \mathbb{C}\right)$ and $H_{*}\left(X_{\gamma_{H}}^{H} ; \mathbb{C}\right)$ are pure. Then there is an isomorphism

$$
H_{m}\left(\Lambda \backslash X_{\gamma} ; \mathscr{L}_{s}\right) \cong H_{m-2 r}\left(\Lambda \backslash X_{\gamma_{H}}^{H} ; \mathscr{M}_{s}\right) .
$$

If the homology of $X_{\gamma}$ is both pure and Tate, and if the same is true of $X_{\gamma_{H}}^{H}$, then this isomorphism may be chosen in a canonical manner.

\subsection{Proof}

The $E^{2}$-term of the Cartan-Leray spectral sequence for the above $\Lambda$-equivariant homology group is

$$
\begin{aligned}
E_{p q}^{2}\left(X_{\gamma} ; \mathbb{C}_{s}\right) & =H_{p}\left(\Lambda ; H_{q}\left(X_{\gamma} ; \mathbb{C}_{s}\right)\right) \\
& =\operatorname{Tor}_{p}^{\mathbb{C}[\Lambda]}\left(H_{q}\left(X_{\gamma}\right), \mathbb{C}_{s}\right) \Longrightarrow H_{p+q}\left(\Lambda \backslash X_{\gamma} ; \mathscr{L}_{s}\right) .
\end{aligned}
$$

By Lemma 2.4, the spectral sequence collapses and gives rise to an isomorphism

$$
H_{m}\left(\Lambda \backslash X_{\gamma} ; \mathscr{L}_{s}\right) \cong \bigoplus_{p+q=m} \operatorname{Tor}_{p}^{\mathbb{C}[\Lambda]}\left(H_{q}\left(X_{\gamma} ; \mathbb{C}\right), \mathbb{C}_{s}\right)
$$

which is canonical if $H_{*}\left(X_{\gamma} ; \mathbb{C}\right)$ is also Tate.

Let $J \subset \mathbb{C}[\Lambda]$ be the multiplicative subset generated by the collection of elements $\left(1-\alpha^{\vee}\right)$ with $\alpha \in \Phi^{+}(G)$ and $s\left(\alpha^{\vee}\right) \neq 1$. The homomorphism $\mathbb{C}[\Lambda] \rightarrow \mathbb{C}_{s}(\ell \mapsto$ $s(\ell)$ ) which gives rise to the local system $\mathscr{L}_{s}$ may be factored as the composition of ring homomorphisms

$$
\mathbb{C}[\Lambda] \rightarrow C[\Lambda]_{J} \rightarrow \mathbb{C}_{s}
$$

in which the second map is defined by

$$
\left(1-\alpha^{\vee}\right)^{-1} \ell \mapsto\left(1-s\left(\alpha^{\vee}\right)\right)^{-1} s(\ell)
$$


for any $\alpha^{\vee} \in \Phi^{\vee}(G, T)-\Phi^{\vee}\left(H, T_{H}\right)$ and any $\ell \in \Lambda$. It is well defined because $s\left(\alpha^{\vee}\right) \neq 1$. For any $\mathbb{C}[\Lambda]$-module $V$, the composition (11.3.2) induces an isomorphism

$$
V \otimes_{\mathbb{C}[\Lambda]} \mathbb{C}_{s} \cong V \otimes_{\mathbb{C}[\Lambda]} \mathbb{C}[\Lambda]_{J} \otimes_{\mathbb{C}[\Lambda]_{J}} \mathbb{C}_{s} \cong V_{J} \otimes_{\mathbb{C}[\Lambda]_{J}} \mathbb{C}_{s}
$$

Taking derived functors gives an isomorphism $\operatorname{Tor}_{p}^{\mathbb{C}[\Lambda]}\left(V, \mathbb{C}_{s}\right) \cong \operatorname{Tor}_{p}^{\mathbb{C}[\Lambda]_{J}}\left(V_{J}, \mathbb{C}_{s}\right)$. Taking $V=H_{q}\left(X_{\gamma} ; \mathbb{C}\right)$ gives an isomorphism

$$
H_{m}\left(\Lambda \backslash X_{\gamma} ; \mathscr{L}_{s}\right) \cong \bigoplus_{p+q=m} \operatorname{Tor}_{p}^{\mathbb{C}[\Lambda]_{J}}\left(H_{q}\left(X_{\gamma} ; \mathbb{C}\right)_{J}, \mathbb{C}_{s}\right)
$$

So Proposition 11.2 follows from Corollary 10.3.

\section{4}

Although we have not constructed the isomorphism (11.2.1) in a canonical way, the isomorphism induced by $\Delta$,

$$
E_{p q}^{2}\left(X_{\gamma} ; \mathbb{C}_{s}\right) \cong E_{p, q-2 r}^{2}\left(X_{\gamma_{H}}^{H} ; \mathbb{C}_{S}\right),
$$

is canonical, and it transforms under $\widetilde{\mathscr{W}}_{\gamma}^{G, H}$ by the sign character $\eta$. It follows that the Lefschetz number of any $w \in \widetilde{\mathscr{W}}_{\gamma}^{G, H}$ on $E_{*}^{2}\left(X_{\gamma} ; \mathbb{C}_{S}\right)$ is equal to that on $E_{*}^{2}\left(X_{\gamma_{H}}^{H} ; \mathbb{C}_{S}\right)$ times the sign $\eta(w)$.

\section{Combinatorial lemmas}

\section{1}

Let $\Lambda$ be a free abelian group of rank one, and let $\mathbb{Q}[\Lambda]$ be its group ring. (The group law in $\Lambda$ is written multiplicatively.) A choice of generator $\alpha^{\vee} \in \Lambda$ determines an isomorphism $\Lambda \cong \mathbb{Z}$. Write $\ell_{a} \in \Lambda$ for the element corresponding to $a \in \mathbb{Z}$. Then multiplication by $\ell_{1}=\alpha^{\vee}$ acts as a "shift operator": $\alpha^{\vee} \ell_{a}=\ell_{a+1}$.

The algebra $\mathbb{Q}\left[\partial_{x}, \partial_{t}\right]$ of differential operators in two variables acts on the algebra $\mathbb{Q}[x, t]$ of polynomials in two variables and hence also on the algebra $\mathbb{Q}[\Lambda] \otimes_{\mathbb{Q}} \mathbb{Q}[x, t]$ with

$$
\operatorname{ker}\left(\partial_{t}\right)=\mathbb{Q}[\Lambda] \otimes_{\mathbb{Q}} \mathbb{Q}[x] .
$$

Fix $d, m \in \mathbb{Z}$ with $d \geq 1$. Define

$$
f_{m, d}=\sum_{m \leq a<b \leq m+d} C_{a b}\left(\ell_{b}-\ell_{a}\right) \otimes((a+b) x-t)^{d-1} \in \mathbb{Q}[\Lambda] \otimes_{\mathbb{Q}} \mathbb{Q}[x, t],
$$

where $C_{a b} \in \mathbb{Z}$ is the integer

$$
C_{a b}=(-1)^{a-b}(a-b)\left(\begin{array}{c}
d \\
a-m
\end{array}\right)\left(\begin{array}{c}
d \\
b-m
\end{array}\right) .
$$


Define $J_{v} \subset \mathbb{Q}[\Lambda] \otimes_{\mathbb{Q}} \mathbb{Q}[x, t]$ to be the span

$$
J_{v}=\sum_{d=1}^{v} \sum_{m \in \mathbb{Z}} \mathbb{Q} f_{m, d} .
$$

LEMMA 12.2

Fix $d, m \in \mathbb{Z}$ with $d \geq 1$. Then

$$
f_{m, d}=(-1)^{d} d !\left(1-\alpha^{\vee}\right)^{d} \ell_{m} \otimes x^{d-1} \in \operatorname{ker}\left(\partial_{t}\right) .
$$

If $v \geq 1$, then

$$
J_{v}=\sum_{d=1}^{v}\left(1-\alpha^{\vee}\right)^{d} \mathbb{Q}[\Lambda] \otimes_{\mathbb{Q}} \mathbb{Q}[x]\left\{\partial_{x}^{d}\right\},
$$

where $\mathbb{Q}[x]\left\{\partial_{x}^{d}\right\}$ denotes the polynomials that are annihilated by $\partial_{x}^{d}$, that is, the polynomials of degree at most $d-1$.

\subsection{Proof}

We use the fact (see $[\mathrm{H}, \S 1.1])$ that for any polynomial $p$,

$$
\sum_{k=0}^{n}(-1)^{k}\left(\begin{array}{l}
n \\
k
\end{array}\right) p(k)= \begin{cases}0 & \text { if } \operatorname{deg} p \leq n-1, \\
(-1)^{n} n ! & \text { if } p(k)=k^{n} .\end{cases}
$$

Since $C_{a b}=-C_{b a}$, we may express $f_{m, d}$ as

$$
\begin{gathered}
\sum_{a=m}^{m+d} \sum_{b=m}^{m+d} C_{a b} \ell_{b} \otimes((a+b) x-t)^{d-1} \\
=\sum_{a=m}^{m+d} \sum_{b=m}^{m+d}\left(\ell_{b} \otimes C_{a b} \sum_{j=0}^{d-1}\left(\begin{array}{c}
d-1 \\
j
\end{array}\right)(a+b)^{j} x^{j}(-1)^{d-1-j} t^{d-1-j}\right) \\
=\sum_{b=m}^{m+d} \sum_{j=0}^{d-1}\left(\ell_{b} \otimes(-1)^{b^{\prime}}\left(\begin{array}{c}
d \\
b-m
\end{array}\right)\left(\begin{array}{c}
d-1 \\
j
\end{array}\right) t^{d^{\prime}}\right. \\
\left.\times \sum_{a=m}^{m+d}(-1)^{a-m}\left(\begin{array}{c}
d \\
a-m
\end{array}\right)(a-b)(a+b)^{j} x^{j}\right),
\end{gathered}
$$

where $b^{\prime}=b+d-1-j-m$ and $d^{\prime}=d-1-j$. By (12.3.1), the innermost sum vanishes unless $j=d-1$, leaving

$$
f_{m, d}=(-1)^{d} d ! \sum_{b=m}^{m+d}\left((-1)^{b-m}\left(\begin{array}{c}
d \\
b-m
\end{array}\right) \ell_{b}\right) \otimes x^{d-1},
$$


which is (12.2.1). To verify the reverse inclusion in equation (12.2.2), it suffices to show that $\left(1-\alpha^{\vee}\right)^{d} \ell_{m} \otimes x^{e-1}$ is in $J_{v}$ for every $d, e$ with $1 \leq e \leq d \leq v$. But

$$
\begin{aligned}
\left(1-\alpha^{\vee}\right)^{d} \ell_{m} \otimes x^{e-1} & =\left(1-\alpha^{\vee}\right)^{e}\left(1-\alpha^{\vee}\right)^{d-e} \ell_{m} \otimes x^{e-1} \\
& =\left(1-\alpha^{\vee}\right)^{e} \sum_{j=0}^{d-e}(-1)^{d-e-j}\left(\begin{array}{c}
d-e \\
j
\end{array}\right) \ell_{m+j} \otimes x^{e-1} \\
& =\frac{(-1)^{d-e}}{e !} \sum_{j=0}^{d-e}(-1)^{j}\left(\begin{array}{c}
d-e \\
j
\end{array}\right) f_{m+j, e}
\end{aligned}
$$

by (12.2.1). Since $e \leq v$, this quantity lies in $J_{v}$. This completes the proof.

\section{LEMMA 12.4}

Fix $d, h, v \geq 1$ with $v \leq h$. Fix $m \in \mathbb{Z}$. Let

$$
g=\sum_{\substack{m \leq a<b \leq m+h \\ b-a \leq b}}\left(\ell_{b}-\ell_{a}\right) \otimes G_{a b}((a+b) x-t)^{d-1},
$$

where $G_{a b} \in \mathbb{Q}$ (with $b>a$ ). If $\partial_{t} g=0$ and $d>v$, then $g=0$.

\subsection{Proof}

Write $g_{a b}=G_{a b}((a+b) x-t)^{d-1}$. The sum in (12.4.1) may be written as either

$$
\sum_{a=m}^{m+h-1} \sum_{b=a+1}^{\min (m+h, a+v)} \text { or } \sum_{b=m+1}^{m+h} \sum_{a=\max (m, b-v)}^{b-1} .
$$

Therefore

$$
\begin{aligned}
g & =\sum_{b=m+1}^{m+h} \sum_{a=\max (m, b-v)}^{b-1} \ell_{b} \otimes g_{a b}-\sum_{a=m}^{m+h-1} \sum_{b=a+1}^{\min (m+h, a+v)} \ell_{a} \otimes g_{a b} \\
& =\sum_{a=m}^{m+h} \ell_{a} \otimes\left(\sum_{b=\max (m, a-v)}^{a-1} g_{b a}-\sum_{b=a+1}^{\min (m+h, a+v)} g_{a b}\right) .
\end{aligned}
$$

(But if $a=m$, the first sum is empty, while if $a=m+h$, the second sum is empty.) Fix $a$ with $m \leq a \leq m+h$. Expanding $((a+b) x-t)^{d-1}$, the equation $\partial_{t} g=0$ gives the system of linear equations

$$
\sum_{b=\max (m, a-v)}^{a-1} G_{b a}(a+b)^{j}-\sum_{b=a+1}^{\min (m+h, a+v)} G_{a b}(a+b)^{j}=0
$$


for $0 \leq j \leq d-2$. This is a system of $d-1$ homogeneous equations of van der Monde type, in $\min (m+h, a+v)-\max (m, a-v)$ variables. If the number of equations equals or exceeds the number of variables, then only the zero solution exists since the corresponding van der Monde determinant is nonzero. Suppose $d>v$. First consider the system (12.5.1) corresponding to $a=m$. Then the first sum in (12.5.1) is empty, leaving the second sum, which consists of $d-1$ (homogeneous) equations (of van der Monde type) in at most $v$ unknowns $G_{m b}$ (with $a+b \leq b \leq a+v$ ). So these coefficients vanish. Suppose by induction that the coefficients $G_{a b}$ have been shown to vanish for all pairs $(a, b)$ with $a<t$ and $a<b \leq \min (m+h, a+v)$. Consider the system (12.5.1) in the case $a=t$. The terms in the first sum, $\sum_{x=\max (m, t-v)}^{t-1} G_{x t}$, vanish by the induction hypothesis, leaving only the second sum. This is a system of $d-1$ homogeneous equations in at most $v$ variables, so only the zero solution $G_{t b}=0$ exists. This completes the induction, so we conclude that $g=0$.

We remark that the same (inductive) argument shows that if $d=v=h$, then there is at most a 1-dimensional space of solutions, so $g$ is a multiple of $f_{m, d}$.

12.6

Define $P_{v} \subset \mathbb{Q}[\Lambda] \otimes_{\mathbb{Q}} \mathbb{Q}[x, t]$ to be the vector space spanned by elements

$$
\left(\ell_{b}-\ell_{a}\right) \otimes g_{a b}((a+b) x-t),
$$

where $g_{a b}$ are polynomials and where $|b-a| \leq v$. Let $P_{v}\left\{\partial_{t}\right\}=P_{v} \cap \operatorname{ker}\left(\partial_{t}\right)$.

PROPOSITION 12.7

Fix $v \geq 1$. Then $P_{v}\left\{\partial_{t}\right\}=J_{v}$; that is,

$\operatorname{ker}\left(\partial_{t}\right) \cap \sum_{|b-a| \leq v} \mathbb{Q}\left(\ell_{b}-\ell_{a}\right) \otimes \mathbb{Q}[(a+b) x-t]=\sum_{d=1}^{v}\left(1-\alpha^{\vee}\right)^{d} \mathbb{Q}[\Lambda] \otimes \mathbb{Q}[x]\left\{\partial_{x}^{d}\right\}$.

\subsection{Proof}

The inclusion $J_{v} \subset P_{v}\left\{\partial_{t}\right\}$ follows from Lemma 12.2, so we only need to verify the reverse inclusion. Let $P_{v, h}$ be the vector subspace of $P_{v}$ spanned by elements (12.6.1) such that each $g_{a b}(z)=G_{a b} z^{h}$ is homogeneous of degree $h$ (with $G_{a b} \in \mathbb{Q}$ ). Set $P_{v, h}\left\{\partial_{t}\right\}=P_{v, h} \cap \operatorname{ker} \partial_{t}$. Then

$$
P_{v}\left\{\partial_{t}\right\}=\sum_{h \geq 0} P_{v, h}\left\{\partial_{t}\right\}
$$

and Lemma 12.4 says that $P_{v, h}\left\{\partial_{t}\right\}=0$ whenever $h \geq v$. So we need to show that $P_{v, d-1}\left\{\partial_{t}\right\} \subset J_{v}$ whenever $d \leq v$. Since both $P_{v}\left\{\partial_{t}\right\}$ and $J_{v}$ are modules over $\mathbb{Q}\left[\partial_{x}\right]$, it suffices to consider the case $d=v$; that is, we must show that $P_{v, v-1}\left\{\partial_{t}\right\} \subset J_{v}$. Let 


$$
g=\sum_{\substack{m \leq a<b \leq m+N \\ b-a \leq v}}\left(\ell_{b}-\ell_{a}\right) \otimes G_{a b}((a+b) x-t)^{v-1} \in P_{v, v-1}\left\{\partial_{t}\right\}
$$

(for some $m, N \in \mathbb{Z}$ ), where $G_{a b} \in \mathbb{Q}$. The expression (12.1.1) for $f_{m, v}$ contains a unique term with $b-a=v$. However, the sum (12.8.1) for $g$ contains only terms with $b-a \leq v$, so by subtracting appropriate multiples of $f_{*, v} \in J_{v} \cap P_{v, v-1}\left\{\partial_{t}\right\}$, we can eliminate all terms with $b-a=v$. In other words, there exists $h \in J_{v} \cap P_{v, v-1}\left\{\partial_{t}\right\}$ such that $g-h \in P_{v-1, v-1}\left\{\partial_{t}\right\}=0$. Therefore $g \in J_{v}$.

\subsection{Remarks}

The lemmas in this section refer to the equivariant homology $H_{*}^{T(\mathbb{C})}\left(X_{\leq v}^{\mathrm{SL}(2)}\right)$ of the affine Springer fiber. Each 1-dimensional orbit $E_{a b} \subset X_{\leq v}^{\mathrm{SL}(2)}$ of the extended torus $\widetilde{T}(\mathbb{C})$ determines a relation in $\mathbb{C}[\Lambda] \otimes \mathbb{C}[x, t]=H_{*}^{\widetilde{T}(\mathbb{C})}(\Lambda)$. For $1 \leq d \leq v$, the sum (12.1.1) corresponds to a "constellation" of (finitely many) 1-dimensional orbits, such that the resulting relation $f_{m, d}$ lies in the subalgebra $\mathbb{C}[\Lambda] \otimes \mathbb{C}[x]=H_{*}^{T(\mathbb{C})}(\Lambda)$. This constellation consists of all the 1-dimensional orbits that are contained in a single irreducible component of $X_{\leq d}^{\mathrm{SL}(2)}$. One may think of this constellation of 1-dimensional orbits as being attached to the single orbit (the "longest one" in the constellation) which joins the lattice points $\ell_{m}$ and $\ell_{m+d}$. So $H_{*}^{T(\mathbb{C})}\left(X_{\leq v}\right)$ is the quotient of $\mathbb{C}[\Lambda] \otimes \mathbb{C}[x]$ by the relations (12.2.1), one for each 1-dimensional $\widetilde{T}(\mathbb{C})$-orbit in $X_{\leq v}$.

\section{Affine flag manifold for $\operatorname{SL}(2)$}

The results in this section are parallel to those of $\S \S 6,7,8$, and 12 , so they are presented without detailed proofs.

\section{1}

Throughout this section we take $G=\operatorname{SL}(2)$. Let $B=\left(\begin{array}{c}* * \\ 0 *\end{array}\right)$ be the standard Borel subgroup of SL(2) with corresponding Iwahori subgroup $I \subset G(\mathfrak{o})$, and let $Y=$ $Y^{\mathrm{SL}(2)}=G(F) / I$ be the affine flag manifold with basepoint $x_{0}=I$. Let $T \subset G$ be the torus of diagonal matrices, with its lattice of translations $\Lambda=\left\{\alpha^{\vee}\left(\varepsilon^{m}\right): m \in \mathbb{Z}\right\}$, where $\alpha^{\vee} \in \Phi^{\vee}$ is the simple coroot determined by $T \subset B$ (cf. §5). The torus $T(\mathbb{C})$ acts on $Y$ with fixed points

$$
\ell_{m}=\left(\begin{array}{cc}
\varepsilon^{m} & 0 \\
0 & \varepsilon^{-m}
\end{array}\right) I \quad \text { and } \quad r_{m}=\left(\begin{array}{cc}
0 & \varepsilon^{m} \\
-\varepsilon^{-m} & 0
\end{array}\right) I
$$

for $m \in \mathbb{Z}$. Let $W=W(G, T)=\left\{1, w_{\alpha}\right\}$ be the Weyl group of $G$. Since the extended affine Weyl group $\widetilde{W}=\Lambda \rtimes W$ acts simply transitively on these fixed points, the choice of basepoint $x_{0}=\ell_{0} \in Y$ determines an identification of $\widetilde{W}$ with this set of 
fixed points, which identifies $\alpha^{\vee}\left(\varepsilon^{m}\right) \in \Lambda$ with $\ell_{m} \in Y$ and identifies the reflection $w_{\alpha}$ with $r_{0} \in Y$. Then $\ell_{a} \ell_{b}=\ell_{a+b}, \ell_{a} r_{b}=r_{a+b}, r_{a} \ell_{b}=r_{a-b}$, and $r_{a} r_{b}=\ell_{a-b}$.

For $m \leq 0$, define

$$
x_{m}=\left(\begin{array}{cc}
1 & \varepsilon^{m} \\
0 & 1
\end{array}\right) I \quad \text { and } \quad y_{m}=\left(\begin{array}{cc}
\varepsilon^{m} & -1 \\
1 & 0
\end{array}\right) I .
$$

Then $x_{0}=\ell_{0}$ is still the basepoint. For notational brevity, put $y_{1}=r_{0}$. Let $\widetilde{T}(\mathbb{C})$ denote the extended torus (5.5.1). If $m \leq-1$ and $s \in \mathbb{Z}$, the $\widetilde{T}(\mathbb{C})$-orbit of $\ell_{s} x_{m}$ is 1-dimensional and it connects the fixed points $\ell_{s}$ and $r_{m+s}$. If $m \leq 0$, the $\widetilde{T}(\mathbb{C})-$ orbit of $\ell_{s} y_{m}$ is 1-dimensional and it connects the fixed points $r_{s}$ and $\ell_{m+s}$. Let $\pi: Y^{\mathrm{SL}(2)} \rightarrow X^{\mathrm{SL}(2)}$ be the natural projection from the affine flag manifold to the affine Grassmannian. It is a $G(F)$-equivariant fibration with fiber isomorphic to $G(\mathbb{C}) / B(\mathbb{C}) \cong \mathbb{C P}^{1}$. It satisfies $\pi\left(\ell_{m}\right)=\pi\left(r_{m}\right)$ for all $m$, and $\pi\left(x_{m}\right)=\pi\left(y_{m}\right)$ for all $m \leq 0$.

For $m \leq 0$, the $T(F)$-orbit of $x_{m} \in Y$ is $(-m)$-dimensional, and it projects isomorphically under $\pi$ to the $T(F)$-orbit of the point $\pi\left(x_{m}\right)$ (which was denoted $x_{m}$ in §6). For $m \leq 1$ the $T(F)$-orbit of $y_{m} \in Y$ is $(1-m)$-dimensional. If $m \leq 0$, it fibers over the $T(F)$-orbit of $\pi\left(y_{m}\right)=\pi\left(x_{m}\right)$ with fiber a 1-dimensional affine space.

Fix a regular element $\gamma \in \mathfrak{t}(\mathfrak{o})$, and let

$$
Y_{\gamma}=\left\{x I \in Y: \operatorname{Ad}\left(x^{-1}\right)(\gamma) \in \operatorname{Lie}(I)\right\}
$$

be the affine Springer fiber corresponding to $\gamma$ in the affine flag manifold $Y$. It is preserved by the extended torus $\widetilde{T}(\mathbb{C})$, and the mapping $\pi: Y_{\gamma} \rightarrow X_{\gamma}$ is surjective. (However, it may fail to be a fibration.) The homology of $Y_{\gamma}$ is pure (see [GKM2]). The paving of $Y$ by affine cells gives rise to a paving of $Y_{\gamma}$ by affine cells. In fact, the intersection of $Y_{\gamma}$ with a single Bruhat cell in $G(F) / I$ is either empty or else it is a single affine cell.

PROPOSITION 13.2

The affine flag manifold $Y$ is the disjoint union of countably many $T(F)$-orbits:

$$
Y=\coprod_{n \leq 0} T(F) \cdot\left\{x_{n} \cup y_{n+1}\right\} .
$$

The affine Springer fiber $Y_{\gamma}$ is the union

$$
Y_{\gamma}=Y_{\leq v}=\bigcup_{n=-v}^{0} T(F) \cdot\left\{x_{n} \cup y_{n+1}\right\},
$$

where $v=\operatorname{val}\left(\alpha^{\prime}(\gamma)\right)$. For each ordered pair of integers $s, t \in \mathbb{Z}$, there is a unique 1-dimensional orbit $E_{s t} \subset Y$ of the extended torus $\widetilde{T}(\mathbb{C})$ which connects the fixed 
points $\ell_{s}$ and $r_{t}$. This accounts for all the 1-dimensional orbits of $\widetilde{T}(\mathbb{C})$ in $Y$. The torus $\widetilde{T}(\mathbb{C})$ acts on the orbit $E_{s t}$ through the affine root $(\alpha, s+t)$. The orbit $E_{s t}$ is contained in $Y_{\gamma}$ if and only if $-v \leq t-s \leq v-1$.

\subsection{Proof}

If $s-t \geq 1$, then $E_{s t}$ is the orbit of the point $\ell_{s} x_{t-s}$. It is contained in $Y_{\gamma}$ if and only if $s-t \leq v$. If $s-t \leq 0$, then $E_{s t}$ is the orbit of the point $\ell_{t} y_{s-t}$. It is contained in $Y_{\gamma}$ if and only if $t-s \leq v-1$.

\section{4}

As in $\S 7$, the coroot $\alpha^{\vee}$ determines isomorphisms $\mathbf{S}(\widetilde{\mathfrak{t}}) \cong \mathbb{C}[x, t]$ and $\mathscr{D}(\widetilde{\mathfrak{t}}) \cong$ $\mathbb{C}\left[\partial_{x}, \partial_{t}\right]$. Fix $\gamma \in \mathfrak{t}(\mathfrak{o})$, and let $v=\operatorname{val}\left(\alpha^{\prime}(\gamma)\right)$. Let $M_{a b}$ be the vector subspace of $\mathbb{C}[\widetilde{W}] \otimes_{\mathbb{C}} \mathbf{S}(\widetilde{\mathfrak{t}})$ which is spanned by elements

$$
\left(\ell_{a}-r_{b}\right) \otimes g_{a b}((a+b) x-2 t),
$$

where $g_{a b}$ are polynomials. Then $M_{a b}$ is a $\mathscr{D}(\widetilde{\mathfrak{t}})$-module. Define $Q_{v}=\sum_{a, b} M_{a b}$ to be the sum of those subspaces $M_{a b}$ such that $-v \leq b-a \leq v-1$. Then the inclusion $\widetilde{W} \subset Y_{\gamma}$ induces a short exact sequence on $T(\mathbb{C})$-equivariant homology,

$$
0 \longrightarrow Q_{v}\left\{\partial_{t}\right\} \longrightarrow \mathbb{C}[\tilde{W}] \otimes_{\mathbb{C}} \mathbf{S}(\mathfrak{t}) \longrightarrow H_{*}^{T(\mathbb{C})}\left(Y_{\gamma}\right) \longrightarrow 0,
$$

where the module of relations $Q_{v}\left\{\partial_{t}\right\}$ denotes the submodule of $Q_{v}$ which is annihilated by the differential operator $\partial_{t}$.

PROPOSITION 13.5

Fix $v \geq 1$. The module $Q_{v}\left\{\partial_{t}\right\}$ of relations is spanned by

$$
\sum_{d=1}^{v}\left(1-\alpha^{\vee}\right)^{d} \mathbb{C}[\widetilde{W}] \otimes \mathbf{S}(\mathfrak{t})\left\{\partial_{x}^{d}\right\}
$$

and

$$
\sum_{d=1}^{v}\left(1-\alpha^{\vee}\right)^{d-1}\left(1-w_{\alpha}\right) \mathbb{C}[\widetilde{W}] \otimes \mathbf{S}(\mathfrak{t})\left\{\partial_{x}^{d}\right\}
$$

\subsection{Proof}

This section is parallel to $§ 12$. Fix $a, b \in \mathbb{Z}$, and fix $d \geq 1$. Define

$$
f_{a, b ; d} \in \mathbb{C}[\tilde{W}] \otimes_{\mathbb{C}} \mathbb{C}[x, t]
$$

to be the element

$$
\sum_{u=a}^{a+d-1} \sum_{v=b}^{b+d-1}(-1)^{u-v}\left(\begin{array}{l}
d-1 \\
u-a
\end{array}\right)\left(\begin{array}{l}
d-1 \\
v-b
\end{array}\right)\left(\ell_{u}-r_{v}\right) \otimes((u+v) x-2 t)^{d-1}
$$


As in $§ 12$, we find that

$$
f_{a, b ; d}=(-1)^{a+b}(d-1) !\left(1-\alpha^{\vee}\right)^{d-1}\left(\ell_{a}-r_{b}\right) \otimes x^{d-1} \in \operatorname{ker}\left(\partial_{t}\right) .
$$

Fix $v \geq 1$. If $1 \leq d \leq v$, then $f_{m, m ; d} \in Q_{v}$ and $f_{m+1, m ; d} \in Q_{v}$. (These are the relations coming from the 1-dimensional orbits in each of the two types of irreducible components of $Y_{\leq d}^{\mathrm{SL}(2)}$; cf. Remark 12.9.) Define $K_{v} \subset \mathbb{C}[\widetilde{W}] \otimes_{\mathbb{C}} \mathbb{C}[x, t]$ to be the span

$$
K_{v}=\sum_{m \in \mathbb{Z}} \sum_{d=1}^{v} \mathbb{C} f_{m, m ; d}+\mathbb{C} f_{m+1, m ; d} .
$$

Then $K_{v}$ is a $\mathscr{D}(\mathfrak{t})=\mathbb{C}\left[\partial_{x}\right]$ module, and equation (13.6.1) implies that $K_{v} \subset Q_{v}\left\{\partial_{t}\right\}$. An argument similar to that of $\$ 12.6$ may be used to show that in fact $K_{v}=Q_{v}\left\{\partial_{t}\right\}$. Since

$$
\begin{aligned}
f_{m, m ; d}+f_{m+1, m, d} & =(d-1) !\left(1-\alpha^{\vee}\right)^{d} \ell_{m} \otimes x^{d-1}, \\
f_{m+1, m+1 ; d}+f_{m+1, m, d} & =(d-1) !\left(1-\alpha^{\vee}\right)^{d} r_{m} \otimes x^{d-1},
\end{aligned}
$$

we see that $K_{v}$ is the sum

$$
\sum_{d=1}^{v}\left(1-\alpha^{\vee}\right)^{d} \mathbb{C}[\tilde{W}] \otimes \mathbf{S}(\mathfrak{t})\left\{\partial_{x}^{d}\right\}+\sum_{d=1}^{v} \mathbb{C} f_{0,0 ; d} .
$$

The second set of relations may be replaced by the less efficient but more symmetric collection (13.5.2) by noting that for any $m \geq 0$,

$$
\begin{gathered}
\sum_{j=-m}^{m-1}\left(f_{j, j ; d}+f_{j+1, j ; d}\right)+f_{m, m ; d}=(d-1) !\left(1-\alpha^{\vee}\right)^{d-1}\left(\ell_{-m}-r_{m}\right) \otimes x^{d-1}, \\
\sum_{j=-m}^{m-1}\left(f_{j+1, j+1 ; d}+f_{j+1, j ; d}\right)-f_{m, m ; d}=(d-1) !\left(1-\alpha^{\vee}\right)^{d-1}\left(r_{-m}-\ell_{m}\right) \otimes x^{d-1} .
\end{gathered}
$$

Since $K_{v}$ is a $\mathbb{C}\left[\partial_{x}\right]$-module, we conclude that for any $1 \leq d \leq v$,

$$
f_{0,0 ; d} \in\left(1-\alpha^{\vee}\right)^{d-1}\left(1-w_{\alpha}\right) \mathbb{C}[\widetilde{W}] \otimes_{\mathbb{C}} \mathbf{S}(\mathfrak{t})\left\{\partial_{x}^{d}\right\} \subset K_{v} .
$$

\subsection{The semisimple rank one case}

As in $\S 8.3$, let $H$ be a connected reductive complex algebraic group of semisimple rank one, let $T \subset B \subset H$ be a Borel pair, let $I \subset H(F)$ be the corresponding Iwahori subgroup, and let $Y^{H}=H(F) / I$ be the affine flag manifold for $H$. Let $\alpha, \alpha^{\vee}$ be the simple positive root and coroot. These determine a reflection $w_{\alpha} \in W$ in the (finite) Weyl group for $H$, a 1-dimensional sublattice $\left\langle\alpha^{\vee}\right\rangle$ in the lattice $\Lambda^{H}$ of translations of 
$T$, and a differential operator $\partial_{\alpha} \in \mathscr{D}(\mathfrak{t})$ of degree 1 . Let $\widetilde{W}=\Lambda \rtimes W$ be the extended affine Weyl group. The mapping $\operatorname{SL}(2) \rightarrow H$ determined by $\alpha$ induces an inclusion $\phi_{\alpha}: Y^{\mathrm{SL}(2)} \rightarrow Y^{H}$. Let $\gamma \in \mathfrak{t}(\mathfrak{o})$ be a regular element, and set $v=\operatorname{val} \alpha^{\prime}(\gamma)$. Then the affine flag manifold for $H$ is the disjoint union

$$
Y^{H}=\coprod_{\ell \in \Lambda^{H} /\left\langle\alpha^{\vee}\right\rangle} \ell \phi_{\alpha}\left(Y^{\mathrm{SL}(2)}\right),
$$

and the affine Springer fiber for $\gamma$ is the disjoint union

$$
Y_{\gamma}^{H}=\coprod_{\ell \in \Lambda^{H} /\left\langle\alpha^{\vee}\right\rangle} \ell \phi_{\alpha}\left(Y_{\leq v}^{\mathrm{SL}(2)}\right),
$$

which we may unambiguously denote by $Y_{\leq \nu}^{H}$. It follows, as in $\S 9.3$, that the $T(\mathbb{C})$ equivariant homology of $Y_{\gamma}^{H}$ is again given by the following.

PROPOSITION 13.8

Suppose that $H$ has semisimple rank one. Fix $T \subset B \subset H$, and fix $v \geq 1$. Then the inclusion of the $T(\mathbb{C})$ fixed points in the affine Springer fiber $Y_{\leq v}^{H}$ induces a short exact sequence

$$
0 \longrightarrow Q_{v}\left\{\partial_{t}\right\} \longrightarrow \mathbb{C}[\tilde{W}] \otimes_{\mathbb{C}} \mathbf{S}(\mathfrak{t}) \longrightarrow H_{*}^{T(\mathbb{C})}\left(Y_{\leq v}^{H}\right) \longrightarrow 0,
$$

where the module of relations $Q_{v}\left\{\partial_{t}\right\}$ is the span of (13.5.1) and (13.5.2).

\section{Homology of affine Springer fibers in the affine flag manifold}

The results in this section are parallel to $\S \S 9$ and 10 , so they are presented without detailed proofs.

\section{1}

As in $\S 5$, let $F=\mathbb{C}((\varepsilon))$, let $G$ be a connected reductive complex algebraic group, let $T \subset B \subset G$ be a Borel pair, and let $I \subset G(F)$ be the corresponding Iwahori subgroup. The affine flag manifold is $Y=Y^{G}=G(F) / I$. Let $\Lambda \subset T(F)$ denote the lattice of translations. Identify each coroot $\alpha^{\vee} \in \Phi^{\vee}(G, T)$ with its image $\alpha^{\vee}(\varepsilon) \in \Lambda$. Set $\widetilde{W}=\Lambda \rtimes W$, where $W=W(G(\mathbb{C}), T(\mathbb{C}))$ is the Weyl group. Each cell in the Bruhat decomposition

$$
Y=\coprod_{w \in \widetilde{W}} I w I / I
$$

contains a unique $T(\mathbb{C})$ fixed point. Hence the choice of basepoint determines a oneto-one correspondence

$$
Y^{T(\mathbb{C})} \cong \widetilde{W}
$$


between the set of fixed points and the extended affine Weyl group, which is compatible with the action of the lattice $\Lambda$ of translations.

For each root $\alpha \in \Phi^{+}$, let $w_{\alpha} \in W$ be its corresponding reflection, let $W_{\alpha}=$ $\left\{1, w_{\alpha}\right\}$, and let $H^{\alpha}$ be the connected reductive group of semisimple rank one which contains $T$ and the root subgroup $U_{\alpha} \subset G$. Let $Y^{\alpha}$ be the affine flag manifold for $H^{\alpha}$. If $u \in Y$ is a $T(\mathbb{C})$ fixed point, then it determines an isomorphism

$$
\phi_{u}: Y^{\alpha} \rightarrow H^{\alpha} \cdot u \subset Y
$$

between $Y^{\alpha}$ and the orbit $H^{\alpha} \cdot u$. It restricts to an isomorphism $Y_{\leq v}^{\alpha} \cong Y_{\gamma} \cap H^{\alpha} \cdot u$ of affine Springer fibers, for any regular element $\gamma \in \mathfrak{t}(\mathfrak{o})$ with $v=\operatorname{val} \alpha^{\prime}(\gamma)$. (The action of $w_{\alpha}$ on $Y^{T(\mathbb{C})}$ preserves the fixed points in the orbit $H^{\alpha} \cdot u$, but it interchanges those of "type $\ell$ " and "type $r$ ".) Define

$$
Z^{\alpha}=\coprod_{u \in W_{\alpha} \backslash W} H^{\alpha} \cdot u=\coprod_{u \in W_{\alpha} \backslash W} \phi_{u}\left(Y^{\alpha}\right) .
$$

The proof of the following lemma is parallel to that of Lemma 5.12.

LEMMA 14.2

Let $\gamma \in \mathfrak{t}(\mathfrak{o})$ be a regular element. Then the union of the 0 - and 1-dimensional orbits in the affine Springer fiber is

$$
\left(Y_{\gamma}\right)_{1}=\bigcup_{\alpha \in \Phi^{+}} Z_{\gamma}^{\alpha}
$$

where $Z_{\gamma}^{\alpha}$ is the intersection

$$
Z_{\gamma}^{\alpha}=Y_{\gamma} \cap Z^{\alpha}=\coprod_{u \in W_{\alpha} \backslash W} \phi_{u}\left(Y_{\leq v}^{\alpha}\right) .
$$

If $\alpha \neq \beta$, then $Z_{\gamma}^{\alpha} \cap Z_{\gamma}^{\beta}=\widetilde{W}$.

Each $\alpha \in \Phi^{+}$corresponds to a degree one differential operator $\partial_{\alpha} \in \mathscr{D}(\mathfrak{t})$, to an element $\alpha^{\vee} \in \Lambda$, and to a reflection $w_{\alpha} \in W$. For $\alpha \in \Phi^{+}$, define the $\mathscr{D}(\mathfrak{t})$-submodule $M_{\alpha, \gamma}$ to be the sum

$$
\begin{aligned}
\sum_{d=1}^{\operatorname{val}\left(\alpha^{\prime}(\gamma)\right)}\left(1-\alpha^{\vee}\right)^{d} \mathbb{C}[\widetilde{W}] \otimes_{\mathbb{C}} \mathbf{S}(\mathfrak{t})\left\{\partial_{\alpha}^{d}\right\} & \\
& +\sum_{d=1}^{\operatorname{val}\left(\alpha^{\prime}(\gamma)\right)}\left(1-\alpha^{\vee}\right)^{d-1}\left(1-w_{\alpha}\right) \mathbb{C}[\widetilde{W}] \otimes_{\mathbb{C}} \mathbf{S}(\mathfrak{t})\left\{\partial_{\alpha}^{d}\right\}
\end{aligned}
$$

Using Proposition 13.8, the argument of $\$ 9.3$ gives the following. 
THEOREM 14.3

Let $\gamma \in \mathfrak{t}(\mathfrak{o})$ be a regular element. Suppose the homology $H_{*}\left(Y_{\gamma} ; \mathbb{C}\right)$ is pure. Then the inclusion $\widetilde{W} \subset Y_{\gamma}$ induces an exact sequence of $\mathscr{D}(\mathfrak{t})$-modules,

$$
0 \longrightarrow \sum_{\alpha \in \Phi^{+}} M_{\alpha, \gamma} \longrightarrow \mathbb{C}[\tilde{W}] \otimes_{\mathbb{C}} \mathbf{S}(\mathfrak{t}) \longrightarrow H_{*}^{T(\mathbb{C})}\left(Y_{\gamma}\right) \longrightarrow 0 .
$$

The group $(\widetilde{W} \rtimes \mathbf{A u t})_{\gamma}$ of (9.4.2) acts on this equivariant homology group, and it restricts to an action on the ordinary homology,

$$
H_{*}\left(Y_{\gamma}\right)=H_{*}^{T(\mathbb{C})}\left(Y_{\gamma}\right)\{\mathscr{I}\},
$$

that is, the subgroup of the equivariant homology which is annihilated by the augmentation ideal $\mathscr{I} \subset \mathscr{D}$.

\subsection{Springer action}

Consider the (regular $\otimes$ trivial) action of $\widetilde{W}$ from the right on

$$
\mathbb{C}[\tilde{W}] \otimes \mathbf{S}(\mathfrak{t}) .
$$

This action preserves each relation $M_{\alpha, \gamma}$ (for $\alpha \in \Phi^{+}$). Assume that the homology $H_{*}\left(Y_{\gamma} ; \mathbb{C}\right)$ is pure. Then the right action of $\mathbb{C}[\tilde{W}]$ passes to an action on $H_{*}^{T(\mathbb{C})}\left(Y_{\gamma}\right)$ which is compatible with the $\mathscr{D}(\mathfrak{t})$-module structure and which commutes with the $(\widetilde{W} \rtimes \mathbf{A u t})_{\gamma}$-action. So it restricts to an action (from the right) of $\widetilde{W}$ on the ordinary homology $H_{*}\left(Y_{\gamma}\right)$. It can be shown that this action coincides with the Springer action defined by Lusztig [Lu] and (using a statement from [KL1] whose proof does not appear in the literature) by Sage [S1]. So Theorem 14.3 gives a "formula" for the Springer action.

\subsection{Endoscopic groups}

There are parallels to $\S \S 10$ and 11 for the affine flag manifold also. As in $\S 10$, suppose that $G$ is adjoint, and let $(H, s)$ be endoscopic data for $G$. Fix compatible Borel pairs $T \subset B \subset G$ and $T_{H} \subset B_{H} \subset H$ with resulting identification $T_{H} \cong T$. The group $H$ has root system $\Phi\left(H, T_{H}\right) \subset \Phi(G, T)$ and (extended) affine Weyl group $\widetilde{W}^{H}=$ $\Lambda \rtimes W^{H} \subset \widetilde{W}$. Let $\gamma \in \mathfrak{t}(\mathfrak{o})$ be a regular element with corresponding Springer fibers $Y_{\gamma}$ and $Y_{\gamma_{H}}^{H}$. Suppose that the homology groups $H_{*}\left(Y_{\gamma} ; \mathbb{C}\right)$ and $H_{*}\left(Y_{\gamma_{H}}^{H} ; \mathbb{C}\right)$ are pure. Let $\Delta$ be the homological transfer factor of (10.1.2), let $r=\operatorname{deg}(\Delta)$, and (as before) let $\eta$ be the sign character that gives the action of $\widetilde{\mathscr{W}}_{\gamma}^{G, H}$ on $\Delta$.

We are going to compare the induced module $H_{*}^{T(\mathbb{C})}\left(Y_{\gamma_{H}}^{H}\right)[-2 r] \otimes_{\mathbb{C}\left[\widetilde{W}^{H}\right]} \mathbb{C}[\widetilde{W}]$ to the module $H_{*}^{T(\mathbb{C})}\left(Y_{\gamma}\right)$. One sees immediately that this induced module is equal to the quotient of $\mathbb{C}[\widetilde{W}] \otimes_{\mathbb{C}} \mathbf{S}(\mathfrak{t})$ by $\sum_{\alpha \in \Phi^{+}(H)} M_{\alpha, \gamma}$. Note that the surjection

$$
1 \otimes \Delta: \mathbb{C}[\tilde{W}] \otimes_{\mathbb{C}} \mathbf{S}(\mathfrak{t}) \rightarrow \mathbb{C}[\tilde{W}] \otimes_{\mathbb{C}} \mathbf{S}(\mathfrak{t})
$$


kills $M_{\alpha, \gamma}$ whenever $\alpha \in \Phi^{+}(G)-\Phi^{+}(H)$.

Let $J \subset \mathbb{C}[\Lambda]$ be the multiplicative subset generated by the elements $1-\alpha^{\vee}$ for $\alpha^{\vee} \in \Phi^{\vee}(G)-\Phi^{\vee}(H)$. We use the action of $\Lambda$ on the Springer fibers to regard their homology groups as modules over the group algebra $\mathbb{C}[\Lambda]$, allowing us to localize the homology groups using $J$. Then we have the following result.

\section{THEOREM 14.6}

Suppose that $H_{*}\left(Y_{\gamma}\right)$ and $H_{*}\left(Y_{\gamma_{H}}^{H}\right)$ are pure. Then the mapping $1 \otimes \Delta$ induces a homomorphism of $\mathscr{D}(\mathfrak{t})$-modules,

$$
H_{*}^{T(\mathbb{C})}\left(Y_{\gamma}\right) \rightarrow H_{*}^{T(\mathbb{C})}\left(Y_{\gamma_{H}}^{H}\right)[-2 r] \otimes_{\mathbb{C}\left[\widetilde{W}^{H}\right]} \mathbb{C}[\widetilde{W}],
$$

and a homomorphism on ordinary homology,

$$
H_{*}\left(Y_{\gamma} ; \mathbb{C}\right) \rightarrow H_{*}\left(Y_{\gamma_{H}}^{H} ; \mathbb{C}\right)[-2 r] \otimes_{\mathbb{C}\left[\widetilde{W}^{H}\right]} \mathbb{C}[\widetilde{W}] .
$$

Both homomorphisms transform under $\eta$ with respect to the left action of $\widetilde{\mathscr{W}}_{\gamma}^{G, H}$ and are equivariant with respect to the right action of $\widetilde{W}$, and both homomorphisms become isomorphisms

$$
\begin{aligned}
H_{*}^{T(\mathbb{C})}\left(Y_{\gamma}\right)_{J} \cong H_{*}^{T(\mathbb{C})}\left(Y_{\gamma_{H}}^{H}\right)_{J}[-2 r] \otimes_{\left.\mathbb{C}_{[} \tilde{W}^{H}\right]} \mathbb{C}[\tilde{W}], \\
H_{*}\left(Y_{\gamma} ; \mathbb{C}\right)_{J} \cong H_{*}\left(Y_{\gamma_{H}}^{H} ; \mathbb{C}\right)_{J}[-2 r] \otimes_{\left.\mathbb{C}_{[} \tilde{W}^{H}\right]} \mathbb{C}[\widetilde{W}]
\end{aligned}
$$

after localizing with respect to $\mathrm{J}$.

\section{An orbital integral}

\subsection{Setup}

Let $k$ be a finite field, and let $\bar{k}$ be an algebraic closure of $k$. Let $F=k((\varepsilon))$ and $L=\bar{k}((\varepsilon))$. Let $\mathfrak{o}$ denote the valuation ring $k[[\varepsilon]]$ of $F$. We write $\sigma$ for the Frobenius automorphism of both $\bar{k} / k$ and $L / F$, and we write $\langle\sigma\rangle$ for the infinite cyclic group generated by $\sigma$. We also choose an algebraic closure $\bar{L}$ of $L$ and write $I$ for $\operatorname{Gal}(\bar{L} / L)$. We write $\bar{F}$ for the algebraic closure of $F$ in $\bar{L}$, and we write $\Gamma$ for the Galois group $\operatorname{Gal}(\bar{F} / F)$.

Let $G$ be a connected reductive group over $F$, and assume that $G$ splits over $L$. Let $K$ be a parahoric subgroup of $G(F)$, let $K_{L}$ be the corresponding $\sigma$-stable parahoric subgroup of $G(L)$, and write $\mathfrak{k}$ and $\mathfrak{k}_{L}$ for the corresponding parahoric subalgebras (of $\mathfrak{g}(F)$ and $\mathfrak{g}(L)$, resp.). Let $X$ be the $k$-ind-scheme associated to $G(L) / K_{L}$. Then $X(\bar{k})=G(L) / K_{L}$ and $X(k)=G(F) / K$.

Let $T$ be a maximal $F$-torus in $G$. Let $S$ denote the maximal unramified subtorus of $T$. Thus the cocharacter group* $X_{*}(S)$ can be identified with $X_{*}(T)^{I}$. 
Let $u$ be a regular element in $\operatorname{Lie}(T)(F)$, and assume that $u$ is integral in the sense that $\alpha(u)$ lies in the valuation ring of $\bar{F}$ for every root $\alpha$ of $T$ in $G$. We write $X_{u}$ for the affine Springer fiber $\left\{x \in X: x^{-1} u x \in \mathfrak{k}_{L}\right\}$ studied by Kazhdan and Lusztig in [KL2]. (We usually write $x^{-1} u x$ rather than $\operatorname{Ad}(x)^{-1} u$.)

For $\mu \in X_{*}(S)$ we write $\varepsilon^{\mu}$ for $\mu(\varepsilon) \in S(L)$. The map $\mu \mapsto \varepsilon^{\mu}$ is $\langle\sigma\rangle$ equivariant and identifies $X_{*}(S)$ with a subgroup of $S(L)$ (and of $T(L)$ ) which we denote by $\Lambda$. The group $T(L)$ centralizes $u$ and therefore acts by left translations on $X_{u}$. The quotient $\Lambda \backslash X_{u}$ of $X_{u}$ by the subgroup $\Lambda$ of $T(L)$ is a nonempty projective scheme of finite type over $k$ (see [KL2]).

\subsection{Normalized Haar measure on $T(F)$}

Recall from $[\mathrm{K} 1, \S 7.6]$ the exact sequence

$$
1 \rightarrow T(L)_{1} \rightarrow T(L) \stackrel{w_{T}}{\longrightarrow} X_{*}(T)_{I} \rightarrow 0 .
$$

Taking invariants under $\langle\sigma\rangle$, we get another exact sequence

$$
1 \rightarrow T(F)_{1} \rightarrow T(F) \rightarrow\left(X_{*}(T)_{I}\right)^{\langle\sigma\rangle} \rightarrow 0,
$$

where $T(F)_{1}:=T(F) \cap T(L)_{1}$. Surjectivity at the right end of this last exact sequence is proved in $[\mathrm{K} 1, \S 7.6]$.

Let $d t$ be the Haar measure on $T(F)$ which gives $T(F)_{1}$ measure 1. The group $\Lambda^{\langle\sigma\rangle}$ of $T(F)$ is discrete and cocompact, so the volume of the quotient $\Lambda^{\langle\sigma\rangle} \backslash T(F)$ is finite. We need to compute this volume (with respect to $d t$ ).

\subsection{Volume computation}

We claim that

$$
\operatorname{vol}_{d t}\left(\Lambda^{\langle\sigma\rangle} \backslash T(F)\right)=\frac{\left|\operatorname{cok}\left[X_{*}(S)_{\Gamma} \rightarrow X_{*}(T)_{\Gamma}\right]\right|}{\left|\operatorname{ker}\left[X_{*}(S)_{\Gamma} \rightarrow X_{*}(T)_{\Gamma}\right]\right|} .
$$

To prove this claim we begin by noting that the canonical map

$$
X_{*}(S)=X_{*}(T)^{I} \rightarrow X_{*}(T)_{I}
$$

has finite kernel and cokernel. Thus this map is in fact injective since $X_{*}(S)$ is torsionfree. We let $M$ denote its cokernel, so that we get a short exact sequence

$$
0 \rightarrow X_{*}(S) \rightarrow X_{*}(T)_{I} \rightarrow M \rightarrow 0
$$

of $\langle\sigma\rangle$-modules, and an associated long exact sequence of group cohomology

$$
\begin{aligned}
0 & \rightarrow X_{*}(S)^{\langle\sigma\rangle} \rightarrow\left(X_{*}(T)_{I}\right)^{\langle\sigma\rangle} \rightarrow M^{\langle\sigma\rangle} \\
& \rightarrow X_{*}(S)_{\Gamma} \rightarrow X_{*}(T)_{\Gamma} \rightarrow M_{\langle\sigma\rangle} \rightarrow 0
\end{aligned}
$$


in which we have identified $X_{*}(S)_{\langle\sigma\rangle}$ with $X_{*}(S)_{\Gamma}$ and $\left(X_{*}(T)_{I}\right)_{\langle\sigma\rangle}$ with $X_{*}(T)_{\Gamma}$.

Since $X_{*}(S)^{\langle\sigma\rangle} \rightarrow\left(X_{*}(T)_{I}\right)^{\langle\sigma\rangle}$ is injective, the subgroups $X_{*}(S)^{\langle\sigma\rangle}$ and $T(F)_{1}$ of $T(F)$ have trivial intersection, and therefore

$$
\operatorname{vol}_{d t}\left(\Lambda^{\langle\sigma\rangle} \backslash T(F)\right)=\left|\frac{\left(X_{*}(T)_{I}\right)^{\langle\sigma\rangle}}{X_{*}(S)^{\langle\sigma\rangle}}\right| .
$$

It follows from (15.3.2) that

$$
\left|\frac{\left(X_{*}(T)_{I}\right)^{\langle\sigma\rangle}}{X_{*}(S)^{\langle\sigma\rangle}}\right|=\frac{\left|M^{\langle\sigma\rangle}\right|}{\left|\operatorname{ker}\left[X_{*}(S)_{\Gamma} \rightarrow X_{*}(T)_{\Gamma}\right]\right|} .
$$

Since all groups in the exact sequence

$$
0 \rightarrow M^{\langle\sigma\rangle} \rightarrow M \stackrel{\sigma-1}{\longrightarrow} M \rightarrow M_{\langle\sigma\rangle} \rightarrow 0
$$

are finite, we have

$$
\left|M^{\langle\sigma\rangle}\right|=\left|M_{\langle\sigma\rangle}\right| .
$$

Finally, it follows from (15.3.2) that

$$
\left|M_{\langle\sigma\rangle}\right|=\operatorname{cok}\left[X_{*}(S)_{\Gamma} \rightarrow X_{*}(T)_{\Gamma}\right] .
$$

Combining the last four equations, we get the claim.

\subsection{Normalized orbital integrals}

For any compactly supported locally constant function $f$ on $\mathfrak{g}(F)$, we put

$$
O_{u}(f)=\int_{T(F) \backslash G(F)} f\left(g^{-1} u g\right) d t \backslash d g,
$$

where $d t$ is the normalized Haar measure on $T(F)$ defined above, and $d g$ is the Haar measure on $G(F)$ that gives our chosen parahoric subgroup $K$ measure 1 . We then have

$$
O_{u}(f)=\operatorname{vol}_{d t}\left(\Lambda^{\langle\sigma\rangle} \backslash T(F)\right)^{-1} \int_{\Lambda^{\langle\sigma\rangle} \backslash G(F)} f\left(g^{-1} u g\right) d g .
$$

In the special case when our function $f$ is $\mathbf{1}_{\mathfrak{k}}$, the characteristic function of $\mathfrak{k}$, we have

$$
\begin{aligned}
\int_{\Lambda^{\langle\sigma\rangle} \backslash G(F)} \mathbf{1}_{\mathfrak{k}}\left(g^{-1} u g\right) d g & =\left|\left\{x \in \Lambda^{\langle\sigma\rangle} \backslash G(F) / K: x^{-1} u x \in \mathfrak{k}\right\}\right| \\
& =\left|\Lambda^{\langle\sigma\rangle} \backslash\left(X_{u}(k)\right)\right| .
\end{aligned}
$$

Combining (15.3.1), (15.4.1), and (15.4.2), we find that

$$
O_{u}\left(\mathbf{1}_{\mathfrak{k}}\right)=\frac{\left|\operatorname{ker}\left[X_{*}(S)_{\Gamma} \rightarrow X_{*}(T)_{\Gamma}\right]\right|}{\left|\operatorname{cok}\left[X_{*}(S)_{\Gamma} \rightarrow X_{*}(T)_{\Gamma}\right]\right|} \cdot\left|\Lambda^{\langle\sigma\rangle} \backslash\left(X_{u}(k)\right)\right| .
$$




\section{5. $\kappa$-orbital integrals of $\mathbf{1}_{\mathfrak{k}}$}

Consider an element $u^{\prime} \in \mathfrak{g}(F)$ which is stably conjugate to $u$ (i.e., conjugate under $G(\bar{F})$ ). Since the group $H^{1}(L, T)$ vanishes (see [Se, §X.7]), there exists $g \in G(L)$ such that $u^{\prime}=g u g^{-1}$. Apply $\sigma$ to this equality to see that $g^{-1} \sigma(g) \in T(L)$. The class of $g^{-1} \sigma(g)$ in $B(T)$ (in other words, the $\sigma$-conjugacy class of $g^{-1} \sigma(g)$ in $\left.T(L)\right)$ is independent of the choice of $g$. Here, as usual, for any linear algebraic group $G$ over $F$, we write $B(G)$ for the set of $\sigma$-conjugacy classes in $G(L)$.

Turning this around, given $t \in T(L)$ whose class in $B(T)$ lies in

$$
\mathfrak{D}(T / F):=\operatorname{ker}[B(T) \rightarrow B(G)],
$$

we choose $g \in G(L)$ such that $t=g^{-1} \sigma(g)$ and put $u(t)=g u g^{-1}$, an element of $\mathfrak{g}(F)$ that is stably conjugate to $u$ and whose $G(F)$-conjugacy class depends only on the class of $t$ in $B(T)$. The construction $t \mapsto u(t)$ sets up a bijection from $\mathfrak{D}(T / F)$ to the set of $G(F)$-conjugacy classes in the stable conjugacy class of $u$.

Recall from $[\mathrm{K} 1, \S 7.6]$ that the canonical homomorphism

$$
w_{T}: T(L) \rightarrow X_{*}(T)_{I}
$$

induces an isomorphism (take coinvariants under $\langle\sigma\rangle$ )

$$
B(T) \simeq X_{*}(T)_{\Gamma} .
$$

At this point we fix a prime number $l$ that is nonzero in the field $k$. Put

$$
\hat{T}:=\operatorname{Hom}\left(X_{*}(T), \overline{\mathbf{Q}}_{l}^{\times}\right) .
$$

Let $\kappa \in \operatorname{Hom}\left(X_{*}(T)_{\Gamma}, \overline{\mathbf{Q}}_{l}^{\times}\right)=\hat{T}^{\Gamma}$. For $t \in T(L)$, we write $\langle t, \kappa\rangle \in \overline{\mathbf{Q}}_{l}^{\times}$for the value of the character $\kappa$ on the element of $X_{*}(T)_{\Gamma}$ obtained as the image of $t$ under

$$
T(L) \rightarrow B(T) \simeq X_{*}(T)_{\Gamma} .
$$

For $\overline{\mathbf{Q}}_{l}$-valued locally constant compactly supported functions $f$ on $\mathfrak{g}(F)$, we define the $\kappa$-orbital integral $O_{u}^{\kappa}(f)$ by

$$
O_{u}^{\kappa}(f)=\sum_{t \in \mathfrak{D}(T / F)}\langle t, \kappa\rangle \cdot O_{u(t)}(f) .
$$

Note that if $\kappa$ lies in the subgroup $Z(\hat{G})^{\Gamma}$ of $\hat{T}^{\Gamma}$ (where we form the Langlands dual group $\hat{G}$ using $\overline{\mathbf{Q}}_{l}$ and where $Z(\hat{G})$ denotes the center of $\left.\hat{G}\right)$, then $O_{u}^{\kappa}$ is a stable orbital integral.

In the special case when $f$ is $\mathbf{1}_{\mathfrak{k}}$, we find that

$$
O_{u}^{\kappa}\left(\mathbf{1}_{\mathfrak{k}}\right)=\frac{|\operatorname{ker}[B(S) \rightarrow B(T)]|}{|\operatorname{cok}[B(S) \rightarrow B(T)]|} \cdot \sum_{t \in \mathfrak{D}(T / F)}\langle t, \kappa\rangle \cdot\left|\Lambda^{\langle\sigma\rangle} \backslash X_{u}^{t \sigma}\right|,
$$


where we have written $X_{u}^{t \sigma}$ for the fixed point set of $t \sigma$ on $X_{u}$, which appears in our formula since $x \mapsto g x$ induces a bijection from $X_{u}^{t \sigma}$ to $X_{u(t)}(k)$, where $g \in G(L)$ is chosen so that $t=g^{-1} \sigma(g)$, as before.

\subsection{Local systems $\mathscr{L}_{\eta}$ on $\Lambda \backslash X_{u}$}

Any finite-dimensional (continuous) $l$-adic representation of $\Lambda \rtimes \mathrm{Gal}(\bar{k} / k)$ (with the topology on $\Lambda$ given by all subgroups of finite index) gives rise to a local system on $\Lambda \backslash X_{u}$ over $k$ (which becomes constant when pulled back to $X_{u}$ over $\bar{k}$ ). Put $\hat{S}:=$ $\operatorname{Hom}\left(X_{*}(S), \overline{\mathbf{Q}}_{l}^{\times}\right)$. Let

$$
\eta \in \operatorname{Hom}\left(X_{*}(S)_{\Gamma}, \overline{\mathbf{Q}}_{l}^{\times}\right)=\hat{S}^{\Gamma}=\hat{S}^{\langle\sigma\rangle},
$$

and assume that $\eta$ has finite order. Using the isomorphism $\Lambda \simeq X_{*}(S)$, we also view $\eta$ as a character on $\Lambda$, and since $\eta$ is fixed by $\sigma$, we may extend $\eta$ to a character on $\Lambda \rtimes \operatorname{Gal}(\bar{k} / k)$ by making it trivial on $\operatorname{Gal}(\bar{k} / k)$. We use the resulting 1-dimensional representation of $\Lambda \rtimes \operatorname{Gal}(\bar{k} / k)$ to form a rank one local system $\mathscr{L}_{\eta}$ on $\Lambda \backslash X_{u}$ over $k$.

Since the action of $T(L)$ on $X_{u}$ commutes with that of $\Lambda$, it induces an action (over $\bar{k}$ ) of $T(L)$ on $\Lambda \backslash X_{u}$ and $\mathscr{L}_{\eta}$, and therefore $T(L)$ acts on the cohomology groups $H^{i}\left(\Lambda \backslash X_{u}, \mathscr{L}_{\eta}\right)$. It follows from the definitions that the subgroup $\Lambda$ of $T(L)$ acts on these cohomology groups through the character $\eta^{-1}$. Moreover, the "identity component" $T(L)_{1}$ of $T(L)$ acts trivially on them. Then since $\Lambda \cdot T(L)_{1}$ has finite index in $T(L)$ and acts by a character on cohomology, each cohomology group is a semisimple $T(L)$-module. Therefore (using (15.2.1)) we can decompose the cohomology groups as

$$
H^{i}\left(\Lambda \backslash X_{u}, \mathscr{L}_{\eta}\right)=\bigoplus_{\kappa} H^{i}\left(\Lambda \backslash X_{u}, \mathscr{L}_{\eta}\right)_{\kappa}
$$

where $\kappa$ runs through the finite set of characters $\kappa \in \operatorname{Hom}\left(X_{*}(T)_{I}, \overline{\mathbf{Q}}_{l}^{\times}\right)=\hat{T}^{I}$ whose image under $\hat{T}^{I} \rightarrow \hat{S}$ is $\eta^{-1}$ and where $H^{i}\left(\Lambda \backslash X_{u}, \mathscr{L}_{\eta}\right)_{\kappa}$ denotes the $\kappa$-isotypic subspace of $H^{i}\left(\Lambda \backslash X_{u}, \mathscr{L}_{\eta}\right)$ (viewing $\kappa$ as a character on $T(L)$, as before).

\subsection{Main result}

Now we can state the main result of this section, which gives a cohomological interpretation of the $\kappa$-orbital integrals of $\mathbf{1}_{\mathfrak{k}}$.

\section{THEOREM 15.8}

Let $\kappa$ be an element of finite order in $\hat{T}^{\Gamma}$. Then the $\kappa$-orbital integral $O_{u}^{\kappa}\left(\mathbf{1}_{\mathfrak{k}}\right)$ is given by

$$
O_{u}^{\kappa}\left(\mathbf{1}_{\mathfrak{k}}\right)=\operatorname{Tr}\left(\sigma^{-1} ; H^{\bullet}\left(\Lambda \backslash X_{u}, \mathscr{L}_{\eta}\right)_{\kappa}\right),
$$

where $\eta$ is the image of $\kappa^{-1}$ under $\hat{T}^{\Gamma} \rightarrow \hat{S}^{\Gamma}$. 
Here we have written $\operatorname{Tr}\left(\sigma^{-1} ; H^{\bullet}\left(\Lambda \backslash X_{u}, \mathscr{L}_{\eta}\right)_{\kappa}\right)$ as an abbreviation for

$$
\sum_{i=0}^{2 \operatorname{dim} X_{u}}(-1)^{i} \operatorname{Tr}\left(\sigma^{-1} ; H^{i}\left(\Lambda \backslash X_{u}, \mathscr{L}_{\eta}\right)_{\kappa}\right) .
$$

\section{Proof}

The first step is to apply the Grothendieck-Lefschetz trace formula. We claim that for any $t \in T(L)$ we have

$$
\operatorname{Tr}\left((t \sigma)^{-1} ; H^{\bullet}\left(\Lambda \backslash X_{u}, \mathscr{L}_{\eta}\right)\right)=\sum_{\bar{x} \in\left(\Lambda \backslash X_{u}\right)^{t \sigma}}\left\langle\lambda_{x}, \eta\right\rangle
$$

with notation as follows. We are writing $\left(\Lambda \backslash X_{u}\right)^{t \sigma}$ for the fixed points of $t \sigma$ on $\Lambda \backslash X_{u}$, and for such a fixed point $\bar{x}$, we choose a representative $x \in X_{u}$ and define $\lambda_{x} \in \Lambda$ by the equality $t \sigma x=\lambda_{x} x$. The image of $\lambda_{x}$ in $\Lambda_{\langle\sigma\rangle}=X_{*}(S)_{\Gamma}$ is independent of the choice of representative $x$. The equality (15.8.1) follows from the GrothendieckLefschetz trace formula applied to the Frobenius map for a twisted ${ }^{*}$-form of $\Lambda \backslash X_{u}$, $\mathscr{L}_{\eta}$ in which the twisted action of $\sigma$ is given by $t \sigma$.

The formula (15.8.1) can be rewritten as

$$
\sum_{\kappa^{\prime}} \operatorname{Tr}\left((t \sigma)^{-1} ; H^{\bullet}\left(\Lambda \backslash X_{u}, \mathscr{L}_{\eta}\right)_{\kappa^{\prime}}\right)=\sum_{\lambda \in X_{*}(S)_{\Gamma}}\langle\lambda, \eta\rangle^{-1} \cdot\left|\Lambda^{\langle\sigma\rangle} \backslash\left(X_{u}^{\varepsilon^{\lambda}} t \sigma\right)\right|,
$$

where the sum on the left is taken over $\kappa^{\prime} \in \hat{T}^{\Gamma}$ whose image under $\hat{T}^{\Gamma} \rightarrow \hat{S}^{\Gamma}$ is $\eta^{-1}$. Indeed, since $(t \sigma)^{-1}$ maps $H^{i}\left(\Lambda \backslash X_{u}, \mathscr{L}_{\eta}\right)_{\kappa^{\prime}}$ to $H^{i}\left(\Lambda \backslash X_{u}, \mathscr{L}_{\eta}\right)_{\sigma^{-1}\left(\kappa^{\prime}\right)}$, only those $\kappa^{\prime} \in \hat{T}^{I}$ that are fixed by $\sigma$ contribute to the trace. As for the sum on the right, we simply collected like terms in the sum on the right in (15.8.1).

Multiply both sides of (15.8.2) by $\langle t, \kappa\rangle$, sum over

$$
t \in \operatorname{cok}[B(S) \rightarrow B(T)]=\operatorname{cok}\left[X_{*}(S)_{\Gamma} \rightarrow X_{*}(T)_{\Gamma}\right],
$$

and divide by the order $\mid$ cok $\mid$ of this cokernel. We obtain

$$
\operatorname{Tr}\left(\sigma^{-1} ; H^{\bullet}\left(\Lambda \backslash X_{u}, \mathscr{L}_{\eta}\right)_{\kappa}\right)=\frac{|\operatorname{ker}|}{|\operatorname{cok}|} \cdot \sum_{t \in B(T)}\langle t, \kappa\rangle \cdot\left|\Lambda^{\langle\sigma\rangle} \backslash\left(X_{u}^{t \sigma}\right)\right| .
$$

Comparing this equation with equation (15.5.1), we see that in order to finish the proof of the theorem, it suffices to show that if $X_{u}^{t \sigma}$ is nonempty, then $t$ lies in $\operatorname{ker}[B(T) \rightarrow$

\footnotetext{
*We are not twisting by a 1-cocycle of $\operatorname{Gal}(\bar{k} / k)$. (Only some elements $t$ yield such a 1-cocycle.) Rather, we are twisting by a 1-cocycle of $\operatorname{Gal}(\bar{k} / k)$ in the group of $\bar{k}$-automorphisms of the projective variety $\Lambda \backslash X_{u}$. Note that we can find a subgroup $A$ of $T(L)$ which acts trivially on $\Lambda \backslash X_{u}$ and for which $T(L) / A$ can be regarded as the $\bar{k}$-points of an algebraic group over $k$, acting algebraically on $\Lambda \backslash X_{u}$.
} 
$B(G)$ ]. Indeed, suppose that $t \sigma(x)=x$ for some $x \in G(L) / K_{L}$. Then $x^{-1} t \sigma(x) \in$ $K_{L}$. By Lang's theorem (applied to the finite-dimensional quotients of the connected proalgebraic group $K_{L}$ over $k$ ) and the completeness of $L$, every element in $K_{L}$ is trivial in $B(G)$. Therefore $t$ is trivial in $B(G)$.

\subsection{A variant of the main result}

The theorem above can be restated in a slightly different way since the classical relationship between the cohomology of $X_{u}$ and that of $\Lambda \backslash X_{u}$ presumably has an $l$-adic version

$$
H^{i}\left(\Lambda \backslash X_{u}, \mathscr{L}_{\eta}\right)_{\kappa}=\operatorname{Ext}_{X_{*}(T)_{I}}^{i}\left(\left(\overline{\mathbf{Q}}_{l}\right)_{\kappa}, R \Gamma\left(X_{u}, \overline{\mathbf{Q}}_{l}\right)\right) .
$$

Here $\left(\overline{\mathbf{Q}}_{l}\right)_{\kappa}$ denotes the 1-dimensional $X_{*}(T)_{I}$-module obtained by letting $X_{*}(T)_{I}$ act on $\overline{\mathbf{Q}}_{l}$ via the surjection $X_{*}(T)_{I} \rightarrow X_{*}(T)_{\Gamma}$ and the character $\kappa$ of $X_{*}(T)_{\Gamma}$, and the complex $R \Gamma\left(X_{u}, \overline{\mathbf{Q}}_{l}\right)$ is being regarded as an object in a suitable derived category of $X_{*}(T)_{I}$-modules. Note that $\sigma$ acts on $X_{*}(T)_{I}$ as well as on $R \Gamma\left(X_{u}, \overline{\mathbf{Q}}_{l}\right)$; it then acts on

$$
\operatorname{Ext}_{X_{*}(T)_{I}}^{i}\left(\left(\overline{\mathbf{Q}}_{l}\right)_{\kappa}, R \Gamma\left(X_{u}, \overline{\mathbf{Q}}_{l}\right)\right)
$$

as well since $\kappa$ is fixed by $\sigma$. Therefore the theorem above presumably yields the equality

$$
O_{u}^{\kappa}\left(\mathbf{1}_{\mathfrak{k}}\right)=\operatorname{Tr}\left(\sigma^{-1} ; R \operatorname{Hom}_{X_{*}(T)_{I}}^{\bullet}\left(\left(\overline{\mathbf{Q}}_{l}\right)_{\kappa}, R \Gamma\left(X_{u}, \overline{\mathbf{Q}}_{l}\right)\right)\right) .
$$

\subsection{A geometric reformulation of the fundamental lemma}

We now assume that $G$ comes from a (necessarily quasi-split) connected reductive group over $k$, which we still denote by $G$. Thus $G$ is unramified over $F$, and the parahoric subgroup $K:=G(\mathfrak{o})$ is a hyperspecial maximal compact subgroup of $G(F)$; we denote the corresponding parahoric subalgebra by $\mathfrak{k}:=\mathfrak{g}(\mathfrak{o})$.

As above, we form all Langlands dual groups using $\overline{\mathbf{Q}}_{l}$. Let $H$ be an endoscopic group for $G$, and let $s$ be the usual element in $Z(\hat{H})^{\Gamma}$, where $Z(\hat{H})$ denotes the center of $\hat{H}$. We write $\mathfrak{h}$ for the Lie algebra of $H$. We assume that $H$ is also unramified, so that it comes from a (quasi-split) group over $k$, which we still denote by $H$. Thus we also have $K_{H}:=H(\mathfrak{o})$ and $\mathfrak{k}_{H}:=\mathfrak{h}(\mathfrak{o})$.

We use a regular nilpotent element in $\mathfrak{g}(\mathfrak{o})$ whose image in $\mathfrak{g}(k)$ is also regular nilpotent in order to form Kostant's section, obtaining, as in [K2], transfer factors $\Delta\left(u_{H}, u\right)$ which for $u$ lying in Kostant's section take the simple value

$$
D_{G}(u) D_{H}\left(u_{H}\right)^{-1}
$$

(in other words, $\Delta_{I V}\left(u_{H}, u\right)$ in the notation of Langlands and Shelstad [LS]). Here $D_{G}$ denotes the usual discriminant function on the Lie algebra. 
Let $u_{H}$ be an integral $G$-regular semisimple element of $\mathfrak{h}(F)$, and let $u$ be an image of $u_{H}$ (in the sense of Langlands and Shelstad [LS]) that is $G(F)$-conjugate to an element in Kostant's section. (This uniquely determines the $G(F)$-conjugacy class of $u$.) We then have affine Springer fibers $X_{u}, X_{u_{H}}^{H}$ for $u, u_{H}$, respectively. Using the dimension formula for affine Springer fibers (conjectured by Kazhdan and Lusztig [KL2] and proved by Bezrukavnikov [B]), we can rewrite the transfer factor as

$$
\Delta\left(u_{H}, u\right)=q^{-r},
$$

where $r:=\operatorname{dim} X_{u}-\operatorname{dim} X_{u_{H}}^{H}$ and $q$ denotes the cardinality of $k$.

We let $T$ denote the centralizer of $u$ in $G$, and we identify $T$ with the centralizer $T_{H}$ of $u_{H}$ in $H$ via the unique admissible isomorphism whose differential maps $u_{H}$ to $u$. Via the canonical injection $Z(\hat{H}) \hookrightarrow \hat{T}$, the element $s \in Z(\hat{H})^{\Gamma}$ determines an element $\kappa \in \hat{T}^{\Gamma}$.

The conjectural fundamental lemma predicts the equality

$$
O_{u_{H}}^{\kappa}\left(\mathbf{1}_{\mathfrak{k}_{H}}\right)=q^{-r} \cdot O_{u}^{\kappa}\left(\mathbf{1}_{\mathfrak{k}}\right),
$$

which in view of (15.9.1) can be rewritten as the equality of

$$
q^{r} \cdot \operatorname{Tr}\left(\sigma^{-1} ; R \operatorname{Hom}_{X_{*}(T)_{I}}^{\bullet}\left(\left(\overline{\mathbf{Q}}_{l}\right)_{\kappa}, R \Gamma\left(X_{u_{H}}^{H}, \overline{\mathbf{Q}}_{l}\right)\right)\right)
$$

and

$$
\operatorname{Tr}\left(\sigma^{-1} ; R \operatorname{Hom}_{X_{*}(T)_{I}}^{\bullet}\left(\left(\overline{\mathbf{Q}}_{l}\right)_{\kappa}, R \Gamma\left(X_{u}, \overline{\mathbf{Q}}_{l}\right)\right)\right) .
$$

We conjecture the stronger statement that

$$
R \operatorname{Hom}_{X_{*}(T)_{I}}^{\bullet}\left(\left(\overline{\mathbf{Q}}_{l}\right)_{\kappa}, R \Gamma\left(X_{u_{H}}^{H}, \overline{\mathbf{Q}}_{l}[-2 r](-r)\right)\right)
$$

is isomorphic to

$$
R \operatorname{Hom}_{X_{*}(T)_{I}}^{\bullet}\left(\left(\overline{\mathbf{Q}}_{l}\right)_{\kappa}, R \Gamma\left(X_{u}, \overline{\mathbf{Q}}_{l}\right)\right) .
$$

\subsection{Transfer factors for unramified tori}

Earlier in this paper, when we calculated the homology of Springer fibers, the elements of $\mathfrak{g}(F)$ we considered did not necessarily lie in Kostant's section. Therefore we need a further discussion of transfer factors.

We continue the discussion from Section 15.10 above, making only the following changes. We no longer require that the elements in $\mathfrak{g}(F)$ that we consider be $G(F)$ conjugate to an element in Kostant's section. Instead we consider a maximal torus $T$ of $G$ over $k$ (not just over $F$ ), so that in particular $T$ is unramified over $F$, and we assume that $T$ comes from a maximal torus $T_{H}$ in $H$. We identify $T$ and $T_{H}$ using some admissible isomorphism, and for any $G$-regular $\gamma \in \mathfrak{t}(F)$, we denote by $\gamma_{H}$ the 
corresponding element of $\mathfrak{t}_{H}(F)$. It follows immediately from the definition of transfer factors on the Lie algebra (see [W] and perhaps also [K2]) that there is a constant $c_{T}$ (depending only on the torus $T$ and our choice of admissible isomorphism) such that for any $G$-regular element $\gamma \in \mathfrak{t}(F)$,

$$
\Delta\left(\gamma_{H}, \gamma\right)=c_{T} \cdot \Delta_{I V}\left(\gamma_{H}, \gamma\right) \cdot \prod_{\alpha}(-1)^{\operatorname{val}(\alpha(\gamma))},
$$

where $\alpha$ runs over a set of representatives for the symmetric orbits of Frobenius on the set of roots of $T$ in $G$ that do not come from $H$. Here* val is the valuation on $\bar{F}$ that takes the value 1 on uniformizing elements for $F$. In fact, the constant $c_{T}$ is equal to 1 since any element $\gamma \in \mathfrak{t}(\mathfrak{o})$ whose image in $\mathfrak{t}(k)$ is $G$-regular (which implies that $\operatorname{val}(\alpha(\gamma))=0$ for all roots $\alpha$ ) is $G(F)$-conjugate to an element in our choice of Kostant's section, and therefore for such $\gamma$ we have (by [K2]) $\Delta\left(\gamma_{H}, \gamma\right)=$ $\Delta_{I V}\left(\gamma_{H}, \gamma\right)$.

Now recall the homological transfer factor $\Delta=\prod_{\alpha \in \Phi^{+}(G)-\Phi^{+}(H)} \partial_{\alpha}^{\mathrm{val} \alpha(\gamma)}$. Frobenius acts on $X^{*}(T)$ by some element $\tau \in \widetilde{\mathscr{W}}_{\gamma}^{G, H}$, and we have $\tau(\Delta)=\eta(\tau) \cdot \Delta$ (see $\S 10$ for the definitions of the group $\widetilde{\mathscr{W}}_{\gamma}^{G, H}$ and the sign character $\eta$ on it). It is a simple exercise to check that

$$
\eta(\tau)=\prod_{\alpha}(-1)^{\operatorname{val}(\alpha(\gamma))}
$$

with $\alpha$ again running over a set of representatives for the symmetric orbits of Frobenius on the set of roots of $T$ in $G$ that do not come from $H$. Therefore we have the equality

$$
\Delta\left(\gamma_{H}, \gamma\right)=\eta(\tau) \cdot \Delta_{I V}\left(\gamma_{H}, \gamma\right),
$$

which for integral $\gamma$ can be rewritten as

$$
\Delta\left(\gamma_{H}, \gamma\right)=\eta(\tau) \cdot q^{-r}
$$

with $r$ again defined as $\operatorname{dim}\left(X_{\gamma}\right)-\operatorname{dim}\left(X_{\gamma_{H}}^{H}\right)$.

\subsection{Remarks on the fundamental lemma for unramified tori}

We continue the discussion of Section 15.11. Assume that the étale cohomology groups $H^{*}\left(X_{\gamma}, \overline{\mathbb{Q}}_{\ell}\right)$ and $H^{*}\left(X_{\gamma_{H}}^{H} ; \overline{\mathbb{Q}}_{\ell}\right)$ are pure. By [GKM2], this is true in the equal valuation case, in other words, when $\operatorname{val}(\alpha(\gamma))$ is independent of $\alpha$, since in that case there is a paving by affines defined over $k$.

We expect Theorems 9.2 and 10.2 to have corresponding statements in étale cohomology. Assume that this is so. Then Theorem 9.2 gives a formula for the $T$ - 
equivariant étale homology of $X_{\gamma}$ as a quotient:

$$
0 \longrightarrow \sum_{\alpha \in \Phi_{G}^{+}} L_{\alpha, \gamma} \longrightarrow \overline{\mathbb{Q}}_{\ell}[\Lambda] \otimes \mathbf{S}\left(\mathfrak{t}\left(\overline{\mathbb{Q}}_{\ell}\right)\right) \longrightarrow H_{*}^{T}\left(X_{\gamma}, \overline{\mathbb{Q}}_{\ell}\right) \longrightarrow 0
$$

The surjection $\overline{\mathbb{Q}}_{\ell}[\Lambda] \otimes \mathbf{S}\left(\mathfrak{t}\left(\overline{\mathbb{Q}}_{\ell}\right)\right) \rightarrow H_{*}^{T}\left(X_{\gamma}, \overline{\mathbb{Q}}_{\ell}\right)$ is Frobenius equivariant, and the action of Frobenius on $\overline{\mathbb{Q}}_{\ell}[\Lambda] \otimes \mathbf{S}\left(\mathfrak{t}\left(\overline{\mathbb{Q}}_{\ell}\right)\right)$ is given by $\tau \otimes Q$, where $\tau \in \widetilde{\mathscr{W}}_{\gamma}^{G, H}$ is the element through which the Frobenius element $\sigma$ acts on $X_{*}(T)$ and $Q$ denotes the endomorphism of the symmetric algebra induced by multiplication by $q$ on the vector space $\mathfrak{t}\left(\overline{\mathbb{Q}}_{\ell}\right)$. It follows that the action of the Frobenius element $\sigma$ on $H_{2 i}\left(X_{\gamma} ; \overline{\mathbb{Q}}_{\ell}\right)$ is given by $\sigma=q^{i} \cdot \tau$.

Therefore Corollary 10.3 gives an isomorphism

$$
H_{*}\left(X_{\gamma} ; \overline{\mathbb{Q}}_{\ell}\right)_{J} \simeq H_{*}\left(X_{\gamma_{H}}^{H} ; \overline{\mathbb{Q}}_{\ell}\right)_{J}[-2 r](r)
$$

which transforms by the sign $\eta(\tau)$ under the action of Frobenius. As in the proof of Proposition 11.2, this in turn gives an isomorphism

$$
\operatorname{Tor}_{p}^{\Lambda}\left(\left(\overline{\mathbb{Q}}_{\ell}\right)_{\kappa}, H_{q}\left(X_{\gamma} ; \overline{\mathbb{Q}}_{\ell}\right)\right) \simeq \operatorname{Tor}_{p}^{\Lambda}\left(\left(\overline{\mathbb{Q}}_{\ell}\right)_{\kappa}, H_{q-2 r}\left(X_{\gamma_{H}}^{H} ; \overline{\mathbb{Q}}_{\ell}\right)\right)(r) .
$$

Taking the vector space dual of both sides of this equation, we obtain an isomorphism

$$
\operatorname{Ext}_{\Lambda}^{p}\left(\left(\overline{\mathbb{Q}}_{\ell}\right)_{\kappa}, H^{q}\left(X_{\gamma} ; \overline{\mathbb{Q}}_{\ell}\right)\right) \simeq \operatorname{Ext}_{\Lambda}^{p}\left(\left(\overline{\mathbb{Q}}_{\ell}\right)_{\kappa}, H^{q-2 r}\left(X_{\gamma_{H}}^{H} ; \overline{\mathbb{Q}}_{\ell}\right)\right)(-r)
$$

which transforms by the sign $\eta(\tau)$ under the action of Frobenius. It follows that

$$
\begin{aligned}
q^{-r} \eta(\tau) \operatorname{Tr}\left(\sigma^{-1} ; R \operatorname{Hom}_{\Lambda}^{\bullet}\left(\left(\overline{\mathbf{Q}}_{l}\right)_{\kappa},\right.\right. & \left.\left.R \Gamma\left(X_{\gamma}, \overline{\mathbf{Q}}_{l}\right)\right)\right) \\
= & \operatorname{Tr}\left(\sigma^{-1} ; R \operatorname{Hom}_{\Lambda}^{\bullet}\left(\left(\overline{\mathbf{Q}}_{l}\right)_{\kappa}, R \Gamma\left(X_{\gamma_{H}}^{H}, \overline{\mathbf{Q}}_{l}\right)\right)\right) .
\end{aligned}
$$

Thus, under the assumptions we have made, the fundamental lemma for unramified tori follows from this last equation together with formulas (15.9.1) and (15.11.1).

Acknowledgments. We wish to thank R. Hain and D. Nadler for useful conversations. We would also like to thank Y. Flicker, G. Lusztig, and B. C. Ngo for their comments on an earlier draft of this paper. The authors are grateful to the University of Chicago, the Université de Paris VII, the École Normale Supérieure, the Institut Henri Poincaré, and the Institute for Advanced Study for their hospitality during various stages of this research.

\section{References}


[CS] T. CHANG and T. SKJELBRED, The topological Schur lemma and related results, Ann. of Math. (2) 100 (1974), 307 - 321. MR 0375357519

[D1] P. DELIGNE, Théorie de Hodge, II, Inst. Hautes Études Sci. Publ. Math. 40 (1971), 5-57. MR 0498551516

[D2] - "Poids dans la cohomologie des variétés algébriques" in Proceedings of the International Congress of Mathematicians (Vancouver, B.C., Canada, 1974), Canad. Math. Congress, Montreal, 1975, 79-85. MR 0432648512

[GKM1] M. GORESKY, R. KOTTWITZ, and R. MACPHERSON, Equivariant cohomology, Koszul duality, and the localization theorem, Invent. Math. 131 (1998), 25 - 83.

MR 1489894 510, 513, 519

[GKM2] — Purity of equivalued affine Springer fibers, preprint, arXiv:math.RT/0305141 $521,528,544,558$

[H] M. HALL, Combinatorial Theory, 2nd ed., Wiley-Intersci. Ser. Discrete Math., Wiley, New York, 1986. MR 0840216540

[Hu] J. E. HUMPHREYS, Conjugacy Classes in Semisimple Algebraic Groups, Math. Surveys Monogr. 43, Amer. Math. Soc., Providence, 1995. MR 1343976534

[KL1] D. KAZHDAN and G. LUSZTIG, A topological approach to Springer's representations, Adv. in Math. 38 (1980), 222-228. MR 0597198 512, 549

[KL2] - Fixed point varieties on affine flag manifolds, Israel J. Math. 62 (1988), 129-168. MR 0947819 510, 520, 521, 538, 551, 557

[K1] R. KOTTWITZ, Isocrystals with additional structure, II, Compositio Math. 109 (1997), 255-339. MR 1485921 551, 553

[K2] _ Transfer factors for Lie algebras, Represent. Theory 3 (1999), 127 - 138. MR 1703328 556, 558

[Ku] S. KUMAR, "An introduction to ind-varieties," Appendix B of "Infinite Grassmannians and moduli spaces of $G$-bundles" in Vector Bundles On Curves-New Directions (Cetraro, Italy, 1995), Lecture Notes in Math. 1649, Springer, Berlin, 1997, 33-38. MR 1605027513

[L] R. P. LANGLANDS, Les débuts d'une formule des traces stables, Publ. Math. Univ. Paris VII 13, Université de Paris VII, U.E.R. de Mathématiques, Paris, 1983. MR 0697567510

[LS] R. P. LANGLANDS and D. SHELSTAD, On the definition of transfer factors, Math. Ann. 278 (1987), 219-271. MR 0909227 556, 557

[La1] G. LAUMON, Fibres de Springer et Jacobiennes compactifiees, preprint, arXiv:math.AG/0204109 512

[La2] - Sur le lemme fondamental pour les groupes unitaires, preprint, arXiv:math.AG/0212245 512

[Lu] G. LUSZTIG, Affine Weyl groups and conjugacy classes in Weyl groups, Transform. Groups 1 (1996), 83-97. MR 1390751 512, 549

[LSm] G. LUSZTIG and J. M. SMELT, Fixed point varieties on the space of lattices, Bull. London Math. Soc. 23 (1991), 213 -218. MR 1123328

[S1] D. S. SAGE, A construction of representations of affine Weyl groups, Compositio Math. 108 (1997), 241 - 245. MR 1473848 512, 549 
[S2] The geometry of fixed point varieties on affine flag manifolds, Trans. Amer. Math. Soc. 352 (2000), 2087 - 2119. MR 1491876

[Se] J.-P. SERRE, Corps Locaux, 2nd ed., Publ. Inst. Math. Univ. Nancago 8, Hermann, Paris, 1968. MR 0354618553

[Sh] I. R. SHAFAREVICH, On some infinite-dimensional groups, II, Math. USSR-Izv. 18 (1982), 185 - 194. MR 0607583513

[W] J.-L. WALDSPURGER, Le lemme fondamental implique le transfert, Compositio Math. 105 (1997), 153-236. MR 1440722558

\section{Goresky}

School of Mathematics, Institute for Advanced Study, 1 Einstein Drive, Princeton, New Jersey 08540, USA; goresky@math.ias.edu

\section{Kottwitz,}

Department of Mathematics, University of Chicago, 5734 South University Avenue, Chicago, Illinois 60637, USA; kottwitz@math.uchicago.edu

\section{MacPherson}

School of Mathematics, Institute for Advanced Study, 1 Einstein Drive, Princeton, New Jersey 08540, USA; rdm@math.ias.edu 